\title{
Advanced fine-tuning of Grubbs/Hoveyda olefin metathesis catalysts: a further step toward an optimum balance between antinomic properties
}

\author{
Michat Bieniek, ${ }^{a}$ Robert Bujok, ${ }^{a}$ Maciej Cabaj, ${ }^{a, b}$ Noël Lugan, ${ }^{c}$ Guy Lavigne,,${ }^{c} *$ Dieter Arlt ${ }^{d, *}$ and \\ Karol Grela ${ }^{a, *}$ \\ ${ }^{a}$ Institute of Organic Chemistry, Polish Academy of Sciences, Kasprzaka 44/52, 01-224, Warsaw (Poland). ${ }^{\mathrm{b}}$ Faculty of \\ Chemistry, Warsaw University of Technology (Politechnika), Noakowskiego 3, 00-664 Warsaw (Poland). ${ }^{\mathrm{c}}$ Laboratoire \\ de Chimie de Cordination du CNRS, 205 route de Narbonne, 31077 Toulouse Cedex 4 (France). ${ }^{\mathrm{d}}$ LIGAND Chemie \\ GmbH, Papenhauser Str. 10, Lemgo, D-32657 (Germany). \\ E-mail: prof.arlt@t-online.de, lavigne@lcc-toulouse.fr, grela@icho.edu.pl
}

\section{Contents}

1 General 2

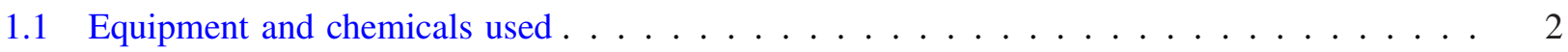

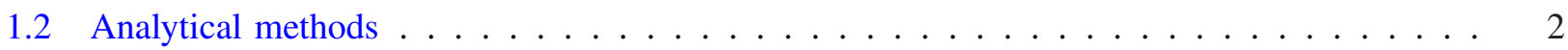

2 Experimental Section $\quad 2$

2.1 Alkylation of 2-propenylphenol. General procedure . . . . . . . . . . . . . . 2

2.1.1 Methyl 2-2-[(1E,Z)-prop-1-en-1-yl]phenoxypropanoate $(\mathbf{3 f}) \ldots \ldots \ldots \ldots$

2.1.2 Methyl 2-4-nitro-2-[(1E,Z)-prop-1-en-1-yl]phenoxypropanoate $(\mathbf{3 g}) \ldots \ldots \ldots$

2.2 General procedure for preparation of catalysts $\mathbf{1 f}$ and $\mathbf{1 g} \ldots \ldots \ldots \ldots$

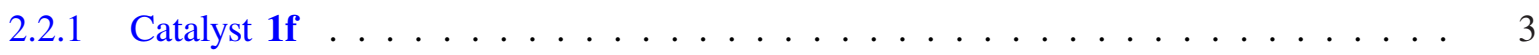

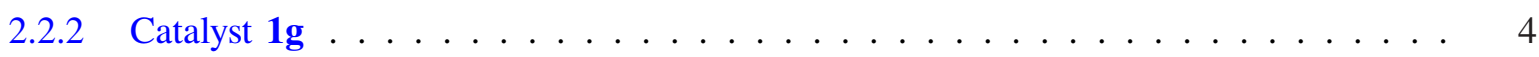

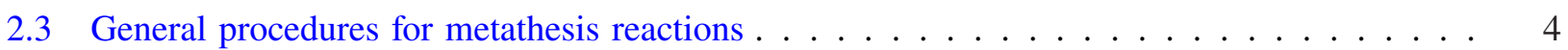

2.3.1 Cross-metathesis . . . . . . . . . . . . . . . . . . . . . . . 4 4

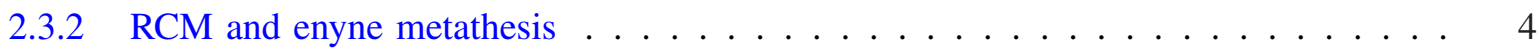

3 Copies of ${ }^{1} \mathrm{H}$ and ${ }^{13} \mathrm{C}-\mathrm{NMR}$ spectra

4 X-ray diffraction $\quad 10$

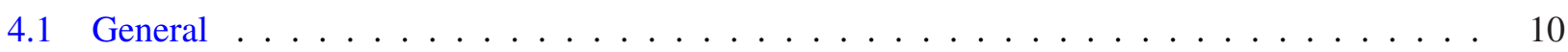

4.2 Molecular view of $\mathbf{1 f}$ in the solid state $\ldots \ldots \ldots \ldots \ldots$

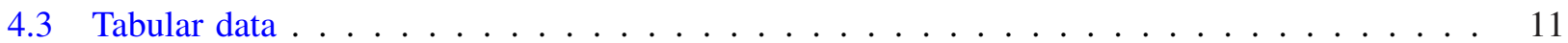




\section{General}

\subsection{Equipment and chemicals used}

The catalyst preparation was carried out under Ar in pre-dried glassware using Schlenk techniques. The anhydrous solvents were dried by distillation over the following drying agents and were transferred under argon: THF (K/benzophenone), toluene (Na), n-pentane, $n$-hexane, $\mathrm{CH}_{2} \mathrm{Cl}_{2}\left(\mathrm{CaH}_{2}\right), \mathrm{Et}_{2} \mathrm{O}\left(\mathrm{LiAlH}_{4}\right), \mathrm{MeOH}$ $(\mathrm{Mg})$.

Flash column chromatography was performed using silica gel 60 (230-400 mesh). NMR spectra were recorded in $\mathrm{CDCl}_{3}$; chemical shifts $(\delta)$ are given in ppm relative to TMS, coupling constants are $(J)$ in Hz. IR spectra: wavenumbers are in $\mathrm{cm}^{-1}$. MS (EI, LSIMS) spectra were recorded on AMD 604 Intectra $\mathrm{GmbH}$ spectrometer. MS (ESI) spectra were recorded on Mariner Perseptive Biosystems, Inc. GC/MS measurements were done on HP 5890 with HP 5 column.

Micro-analyses were provided by Institute of Organic Chemistry, PAS, Warsaw.

Catalysts 1a and 1c were purchased from Aldrich and used as received. Complexes $\mathbf{1 d},{ }^{2} \mathbf{1 e}{ }^{3}$ and ligand precursor $\mathbf{2} \mathbf{g}^{4}$ were prepared according to literature methods. All other commercially available chemicals were used as received.

\subsection{Analytical methods}

Comparative RCM experiments with diene $\mathbf{4 b}$ (refer to Figure 2) were performed as described by Piers et al. ${ }^{5}\left(\mathrm{CH}_{2} \mathrm{Cl}_{2},[\mathbf{4 b}]=0.02 \mathrm{M}, 0{ }^{\circ} \mathrm{C}, 6 \mathrm{~h}\right)$. To a stirred cold solution of diene $4 \mathbf{b}(0.50 \mathrm{mmol}, 120.2 \mathrm{mg})$ and n-nonane (used as an internal standard, $0.25-0.30 \mathrm{mmol}$ ) in $\mathrm{CH}_{2} \mathrm{Cl}_{2}(25 \mathrm{~mL})$ placed under argon in a Schlenk tube a catalyst $(0.005 \mathrm{mmol})$ was added in a single portion at $0 \pm 0.3{ }^{\circ} \mathrm{C}$ and the reaction mixture was stirred for $6 \mathrm{~h}$ at the same temperature. Aliquots $(0.25 \mathrm{~mL})$, taken in regular intervals, were quenched immediately with ice-cold solution of ethyl-vinyl ether $\left(0.25 \mathrm{~mL}, 2 \mathrm{M}\right.$ in $\left.\mathrm{CH}_{2} \mathrm{Cl}_{2}\right)$ and analysed by $\mathrm{GC}$, using HP 6890 chromatograph with HP 5 column. The responses of the FID detector were calibrated using $4 \mathbf{b} / n$-nonane and $5 \mathbf{b} / n$-nonane standard solutions. Each RCM experiment was repeated at least two times.

For the comparative experiments presented in Table 1, the responses of the FID detector were calibrated using $\mathbf{4 a} / n$-nonane, $\mathbf{4 b} / n$-nonane, $\mathbf{5 b} / n$-nonane and $\mathbf{4} \mathbf{c} / n$-nonane standard solutions, respectively (entries $1-3$ ). For the preparative experiments (entries 5-8) the GC FID responses were not calibrated.

\section{Experimental Section}

\subsection{Alkylation of 2-propenylphenol. General procedure}

To a suspension of $\mathrm{K}_{2} \mathrm{CO}_{3}(5.5 \mathrm{~g} ; 40.0 \mathrm{mmol})$ and $\mathrm{Cs}_{2} \mathrm{CO}_{3}(2.6 \mathrm{~g} ; 6.0 \mathrm{mmol})$ in DMF (100 ml), 2propenylphenol $(2.7 \mathrm{~g} ; 20.0 \mathrm{mmol})$ was added. After stirring for $30 \mathrm{~min}$ at room temperature, methyl 2-bromopropionate $(0.8 \mathrm{~mL}, 1.36 \mathrm{~g}, 8.0 \mathrm{mmol})$ was added and the reaction mixture was stirred $1-2$ days at $40{ }^{\circ} \mathrm{C}$. The reaction mixture was poured into $40 \mathrm{ml}$ of water and extracted three times with diethyl ether. The combined extracts were washed with brine, water and dried. The solvent was evaporated to give the crude product $3 \mathbf{f}(3.8 \mathrm{~g} ; 84 \%)$ as a yellow oil. This product was used without further purification.

\subsubsection{Methyl 2-2-[(1E,Z)-prop-1-en-1-yl]phenoxypropanoate (3f)}

Yellow oil (84\%). Mixture of isomers, $E: Z=5: 2$. IR (film): $\nu$ 3032, 2991, 2954, 2914, 1760, 1738, 1598, 1580, 1486, 1450, 1375, 1281, 1236, 1207, 1136, 1051, 975, 794, 749, $605 \mathrm{~cm}^{-1}$. Isomer $E .{ }^{1} \mathrm{H}$ NMR

\footnotetext{
${ }^{2}$ (a) Wakamatsu, H.; Blechert, S. Angew. Chem., Int. Ed. Engl. 2002, 41, 2403. (b) Dunne, A. M.; Mix, S.; Blechert, S. Tetrahedron Lett. 2003, 44, 2733 .

${ }^{3}$ (a) Grela, K.; Harutyunyan; S., Michrowska, A. Angew. Chem. Int. Ed. 2002, 41, 4038. (b) Michrowska, A.; Bujok, R.; Harutyunyan, S.; Sashuk, V.; Dolgonos, G.; Grela, K. J. Am. Chem. Soc. 2004, 126, 9318.

${ }^{4}$ Bujok, R.; Bieniek, M.; Masnyk, M.; Michrowska, A.; Sarosiek, A.; Stępowska, H.; Arlt, D.; Grela, K. J. Org. Chem. 2004, 69, 6894.

${ }^{5}$ Romero, P. E.; Piers, W. E.; McDonald, R. Angew. Chem., Int. Ed. 2004, 43, 6161.

Supplementary data for $10.1021 / \mathrm{ja} 063186 \mathrm{w}$ 
$\left(400 \mathrm{MHz}, \mathrm{CDCl}_{3}\right) \delta 1.65(\mathrm{~d}, J=6.7 \mathrm{~Hz}, 3 \mathrm{H}), 1.90(\mathrm{dd}, J=6.6,1.8 \mathrm{~Hz}, 3 \mathrm{H}), 3.74(\mathrm{~s}, 3 \mathrm{H}), 4.75(\mathrm{q}, J=$ $6.7 \mathrm{~Hz}, 1 \mathrm{H}), 6.70(\mathrm{dd}, J=8.2,1.0 \mathrm{~Hz}, 1 \mathrm{H}), 6.78(\mathrm{dd}, J=16.0,1.8 \mathrm{~Hz}, 1 \mathrm{H}), 6.74(\mathrm{dd}, J=1.8,16.0 \mathrm{~Hz}$, $1 \mathrm{H}), 6.90-6.95(\mathrm{~m}, 1 \mathrm{H}), 7.11(\mathrm{ddd}, J=8.2,7.5,1.7 \mathrm{~Hz}, 1 \mathrm{H}), 7.42(\mathrm{dd}, J=7.7,1.7 \mathrm{~Hz}, 1 \mathrm{H}) \mathrm{ppm}$. Isomer E. ${ }^{13} \mathrm{C}$ NMR $\left(100 \mathrm{MHz}, \mathrm{CDCl}_{3}\right) \delta 18.6,18.9,52.2$, 73.3, 113.0, 121.7, 125.4, 126.6, 127.6, 127.9, 130.4, 154.2, 172.7. MS (EI) $\mathrm{m} / z$ (rel intensity) 221 (11), 220 (77, $\mathrm{M}^{+\cdot}$ ), 161 (54), 145 (13), 134 (25), 133 (100), 131 (23), 119 (52), 115 (37), 105 (82), 91 (49), 79 (17), 77 (27), 59 (16), 51 (13), 43 (22). HRMS (EI) calcd for $\mathrm{M}^{+\cdot}\left(\mathrm{C}_{13} \mathrm{H}_{16} \mathrm{O}_{3}\right)$ : 220.10994; found: 220.11005 Anal. calcd for $\mathrm{C}_{13} \mathrm{H}_{16} \mathrm{O}_{5}$ (220.27): C 70.89, $\mathrm{H}$ 7.32; found C 70.46, H 7.38.

\subsubsection{Methyl 2-4-nitro-2-[(1E,Z)-prop-1-en-1-yl]phenoxypropanoate (3g)}

Yellow oil (89 \%). Mixture of isomers, $E: Z=9: 1$. IR (film): $\nu$ 3083, 2994, 2956, 2852, 1757, 1653, 1584, $1518,1485,1448,1343,1255,1212,1148,1129,1098,1050,973,817,747,657 \mathrm{~cm}^{-1}$. Isomer $E .{ }^{1} \mathrm{H}$ NMR $\left(400 \mathrm{MHz}, \mathrm{CDCl}_{3}\right) \delta 1.72(\mathrm{~d}, J=6.7 \mathrm{~Hz}, 3 \mathrm{H}), 1.95(\mathrm{dd}, J=6.7,1.7 \mathrm{~Hz}, 3 \mathrm{H}), 3.78(\mathrm{~s}, 3 \mathrm{H}), 4.89$ $(\mathrm{q}, J=6.7 \mathrm{~Hz}, 1 \mathrm{H}), 6.41(\mathrm{dq}, J=16.0,6.7 \mathrm{~Hz}, 1 \mathrm{H}), 6.71(\mathrm{~d}, J=9.2 \mathrm{~Hz}, 1 \mathrm{H}), 6.74(\mathrm{dd}, J=1.8,16.0 \mathrm{~Hz}$, $1 \mathrm{H}), 8.02(\mathrm{dd}, J=2.7,9.0 \mathrm{~Hz}, 1 \mathrm{H}), 8.32(\mathrm{~d}, J=2.7 \mathrm{~Hz}, 1 \mathrm{H}) \mathrm{ppm} .{ }^{13} \mathrm{C}$ NMR $\left(100 \mathrm{MHz}, \mathrm{CDCl}_{3}\right) \delta 18.4$, $18.9,52.6,73.2,111.6,122.1,123.3,123.6,128.5,129.8,142.1,158.6,171.4$. MS (EI) $\mathrm{m} / \mathrm{z}$ (rel intensity) 266 (15), 265 (97, M+· ), 248 (6), 207 (11), 206 (87), 190 (8), 178 (34), 164 (48), 161 (18), 160 (29), 145 (23), 132 (100), 131 (35), 118 (24), 115 (22), 103 (15), 88 (12), 77 (17), 59 (23), 43 (18), 39 (7). Anal. calcd for $\mathrm{C}_{13} \mathrm{H}_{15} \mathrm{O}_{5} \mathrm{~N}$ (265.27): C 58.86, H 5.70, N 5.28; found C 58.83, H 5.63, N 5.24.

\subsection{General procedure for preparation of catalysts $1 \mathrm{f}$ and $1 \mathrm{~g}$}

Ligand 3f (54.6 mg; $0.25 \mathrm{mmol}), \mathrm{CuCl}(24.9 \mathrm{mg} ; 0.25 \mathrm{mmol})$ and $\mathrm{CH}_{2} \mathrm{Cl}_{2}(10 \mathrm{~mL})$ were placed in a Schlenk flask. Afterwards carbene complex 1a $(170.9 \mathrm{mg} ; 0.20 \mathrm{mmol})$ was added and the resulting solution was stirred under argon at $40^{\circ} \mathrm{C}$ for $20 \mathrm{~min}$. From this point forth, all manipulations were carried out in air with reagent-grade solvents. The reaction mixture was concentrated under vacuum and the resulting material was dissolved in EtOAc (ca. $20 \mathrm{~mL}$ ), white solid was filtered off and the filtrate was concentrated under vacuum. The product was purified by column chromatography on silica. Elution with $c$-hexane (ca $200 \mathrm{~mL}$ ), then $c$-hexane-EtOAc (2:1) removes $\mathbf{3 f}$ as a green band. The solvent was evaporated and product dissolved in a small amount $\mathrm{CH}_{2} \mathrm{Cl}_{2}$, then $n$-pentane was added until green crystals precipitated. The precipitate was filtered off, washed with $n$-pentane and dried in vacuo to afford complex $\mathbf{1 f}(96.3 \mathrm{mg}, 71 \%)$ as a green solid.

\subsubsection{Catalyst 1f}

Green microcrystalline solid (71 \%). ${ }^{6} \mathrm{IR}(\mathrm{KBr}): \nu$ 3448, 2952, 2918, 2857, 2736, 1932, 1764, 1736, 1667, 1593, 1574, 1479, 1452, 1417, 1398, 1379, 1293, 1263, 1211, 1158, 1131, 1117, 1089, 1037, 975, 936, $918,851,795,745,646,617,579,472,438,422 \mathrm{~cm}^{-1} .{ }^{1} \mathrm{H}$ NMR $\left(500 \mathrm{MHz}, \mathrm{CDCl}_{3}\right) \delta 1.50(\mathrm{~d}, J=6.8$ $\mathrm{Hz}, 3 \mathrm{H}), 2.38(\mathrm{bs}, 6 \mathrm{H}), 2.48(\mathrm{bs}, 12 \mathrm{H}), 3.62(\mathrm{~s}, 3 \mathrm{H}), 4.15(\mathrm{~s}, 4 \mathrm{H}), 4.97(\mathrm{k}, J=6.8 \mathrm{~Hz}, 1 \mathrm{H}), 6.62(\mathrm{~d}, J$ $=8.2 \mathrm{~Hz}, 1 \mathrm{H}), 6.91-6.97(\mathrm{~m}, 3 \mathrm{H}), 7.07(\mathrm{~s}, 4 \mathrm{H}), 7.44-7.49(\mathrm{~m}, 1 \mathrm{H}), 15.58(\mathrm{~s}, 1 \mathrm{H}) \mathrm{ppm} .{ }^{13} \mathrm{C} \mathrm{NMR}(125$ $\left.\mathrm{MHz}, \mathrm{CDCl}_{3}\right) \delta 17.4,21.1,51.6,52.9,73.7,112.3,122.8,123.7,129.0,129.4,138.4,145.4,151.5,171.0$, 210.5, 299.0. MS (EI) $\mathrm{m} / \mathrm{z}$ (rel intensity) 675 (2), 674 (6), 673 (6), 672 (17), 671 (10), 670 (18, $\mathrm{M}^{+\cdot}$ ), 669 (11), 668 (7), 667 (5), 666 (2), 406 (12), 405 (11), 404 (12), 392 (14), 391 (9), 390 (12), 308 (10), 307 (43), 306 (10), 305 (49), 304 (100), 303 (82), 301 (12), 289 (19), 287 (14), 194 (14), 163 (35), 158 (18), 148 (15), 146 (14), 145 (11), 144 (10), 135 (25), 119 (11), 108 (11), 107 (13), 91 (30), 77 (15), 57 (25), 43 (14), 41 (13), 36 (25). HRMS (EI) calcd for $\mathrm{M}^{+\cdot}\left(\mathrm{C}_{32} \mathrm{H}_{38} \mathrm{~N}_{2} \mathrm{O}_{3} \mathrm{Cl}_{2} \mathrm{Ru}\right)$ : 670.13030; found: 670.13467. Anal. calcd for $\mathrm{C}_{13} \mathrm{H}_{15} \mathrm{O}_{5} \mathrm{~N}$ (265.27): C 57.31, H 5.71, N 4.18, Cl 10.57; found C 57.53, H 5.67, N 3.95, Cl 10.48 .

\footnotetext{
${ }^{6}$ A sample of $\mathbf{1 f}$ was dissolved in a small amount EtOAc and left in open flask overnight at room temperature to give crystalls suitable for X-ray analysis. 


\subsubsection{Catalyst $1 \mathrm{~g}$}

Green microcrystalline solid (69 \%). IR (KBr): $\nu$ 3453, 2953, 2917, 2858, 2737, 1737, 1607, 1575, 1524, 1481, 1418, 1379, 1342, 1263, 1231, 1136, 1109, 1089, 1037, 907, 849, 827, 744, 579, $423 \mathrm{~cm}^{-1} .{ }^{1} \mathrm{H} \mathrm{NMR}$ $\left(500 \mathrm{MHz}, \mathrm{CDCl}_{3}\right) \delta 1.53(\mathrm{~d}, J=6.8 \mathrm{~Hz}, 3 \mathrm{H}), 2.40-2.52(\mathrm{~m}, 18 \mathrm{H}), 3.64(\mathrm{~s}, 3 \mathrm{H}), 4.18(\mathrm{~s}, 4 \mathrm{H}), 5.05(\mathrm{q}, J$ $=6.8 \mathrm{~Hz}, 1 \mathrm{H}), 6.74(\mathrm{~d}, J=9.0 \mathrm{~Hz}, 1 \mathrm{H}), 7.09(\mathrm{~s}, 4 \mathrm{H}), 7.83(\mathrm{~d}, J=2.6 \mathrm{~Hz}, 1 \mathrm{H}), 8.41(\mathrm{dd}, J=9.0,2.6 \mathrm{~Hz}$, $1 \mathrm{H}), 16.49$ (s, 1H) ppm. ${ }^{13} \mathrm{C}$ NMR $\left(125 \mathrm{MHz}, \mathrm{CDCl}_{3}\right) \delta 17.3,21.0,51.6,53.2,74.8,112.5,117.3,123.5$, 1129.5, 138.9, 144.2, 144.9, 155.0, 170.0, 207.4, 293.3. MS (EI) $\mathrm{m} / \mathrm{z}$ (rel intensity) 717 (5), $715\left(6, \mathrm{M}^{+\cdot}\right.$ ), 474 (7), 305 (15), 304 (44), 303 (39), 239 (18), 194 (30), 192 (13), 180 (36), 166 (57), 146 (19), 120 (16), 91 (13), 87 (34), 65 (35), 63 (100), 59 (99), 55 (27), 50 (31), 43 (29), 41 (17), 39 (11). HRMS (EI) calcd for $\mathrm{M}^{+\cdot}\left(\mathrm{C}_{32} \mathrm{H}_{37} \mathrm{~N}_{3} \mathrm{O}_{5} \mathrm{Cl}_{2} \mathrm{Ru}\right)$ : 715.11537; found: 715.11683 Anal. calcd for $\mathrm{C}_{32} \mathrm{H}_{37} \mathrm{~N}_{3} \mathrm{O}_{5} \mathrm{Cl}_{2} \mathrm{Ru}$ (715.64): C 53.71, H 5.21, N 5.87, Cl 9.91; found C 53.11, H 5.13, N 5.83, Cl 9.83.

\subsection{General procedures for metathesis reactions}

\subsubsection{Cross-metathesis}

To a mixture of alkene $(0.5 \mathrm{mmol})$ and cross-metathesis partner $(1.0-2.0 \mathrm{mmol})$ in $\mathrm{CH}_{2} \mathrm{Cl}_{2}(25 \mathrm{~mL}, c=$ $0.02 \mathrm{M})$ was added a Ru-catalyst as a solid $(0.0015-0.0150 \mathrm{mmol}, 0.3-3.0 \mathrm{~mol} \%)$. The resulting mixture was stirred at $25-28{ }^{\circ} \mathrm{C}$ for $0.5-16 \mathrm{~h}$. The solvent was removed under reduced pressure. The crude product was purified by flash chromatography $(c$-hexane-EtOAc).

\subsubsection{RCM and enyne metathesis}

To a mixture of alkene $(0.5 \mathrm{mmol})$ in $\mathrm{CH}_{2} \mathrm{Cl}_{2}(25 \mathrm{~mL}, c=0.02 M)$ was added a solution of a Ru-catalyst (0.00015-0.00500 mmol, 0.03-1.00 mol\%). The resulting mixture was stirred at $0-25^{\circ} \mathrm{C}$ for $0.5-6 \mathrm{~h}$. The solvent was removed under reduced pressure. The crude product was purified by flash chromatography (c-hexane-EtOAc).

\section{Methyl 7-[1-(tert-butyl)-1,1-dimethylsilyl]oxy-2-heptenoate (5c)}

Colourless oil. $^{7}$ Mixture of isomers $E: Z=97: 3$. IR (film): $\nu$ 2952, 2933, 2859, 1729, 1659, 1472, 1437 , $1389,1317,1258,1201,1165,1102,1040,983,838,777 \mathrm{~cm}^{-1}$. Isomer $E:{ }^{1} \mathrm{H}$ NMR $\left(500 \mathrm{MHz}, \mathrm{CDCl}_{3}\right) \delta$ $0.03(\mathrm{~s}, 6 \mathrm{H}) 0.88(\mathrm{~s}, 9 \mathrm{H}) 1.46-1.57(\mathrm{~m}, 4 \mathrm{H}) 2.17-2.25(\mathrm{~m}, 2 \mathrm{H}) 3.61(\mathrm{t}, J=5.9 \mathrm{~Hz}, 2 \mathrm{H}) 3.71(\mathrm{~s}, 3 \mathrm{H}) 5.81$ $(\mathrm{dt}, J=15.7,1.6 \mathrm{~Hz}, 1 \mathrm{H}) 6.96(\mathrm{dt}, J=15.7,7.0 \mathrm{~Hz}, 1 \mathrm{H}) \mathrm{ppm}$. Isomer $E:{ }^{13} \mathrm{C} \mathrm{NMR}\left(125 \mathrm{MHz}, \mathrm{CDCl}_{3}\right) \delta$ $-5.3,18.3,24.4,25.9,31.9,32.2,51.3,62.7,121.0,149.4,167.1 \mathrm{ppm}$. MS (EI) $\mathrm{m} / \mathrm{z}$ (rel intensity) 257 (1, $[\mathrm{M}-15]^{+}$) 241 (3) 217 (4) 215 (23) 183 (15) 171 (0,5) 155 (1) 139 (3) 119 (3) 101 (3) 89 (100) 81 (53) 79 (13) 75 (32) 73 (25) 59 (24) 47 (11) 41 (21) 39 (10) HRMS (ESI): calcd for [M+Na] ${ }^{+}\left(\mathrm{C}_{14} \mathrm{H}_{28} \mathrm{O}_{3} \mathrm{SiNa}\right)$ : 295.1700; found: 295.1691.

\section{1-Acetyl-2,5-dihydro-1 $H$-pyrrole $(5 \mathrm{~g})$}

Colorless oil. ${ }^{8}$ IR (film): $\nu$ 3286, 2933, 2866, 1729, 1638, 1615, 1455, 1424, 1376, 1300, 1223, 1063, 986, $856 \mathrm{~cm}^{-1}{ }_{1}^{1} \mathrm{H}$ NMR $\left(400 \mathrm{MHz}, \mathrm{CDCl}_{3}\right) \delta 2.09$ (s, 3H) 4.20-4.29 (m, 4H) 5.77-5.83 (m, 1H) 5.85-5.91 $(\mathrm{m}, 1 \mathrm{H}){ }^{13} \mathrm{C}$ NMR $\left(100 \mathrm{MHz}, \mathrm{CDCl}_{3}\right) \delta 22.3,52.8,54.1,124.8,126.3,167.8$, MS (EI) $\mathrm{m} / z$ (rel intensity) $111\left(48,[\mathrm{M}]^{+\cdot}\right.$ ) 109 (33) 97 (8) 83 (5) 69 (33) 68 (100) 67 (92) 56 (9) 55 (6) 43 (79) 42 (8) 41 (35) 40 (13) 39 (28) 38 (8), HRMS (EI): calcd for [M] $]^{+\cdot}\left(\mathrm{C}_{6} \mathrm{H}_{9} \mathrm{NO}\right)$ : 111.0684; found 111.0685.

2,2-Diphenyl-3-vinyl-2,5-dihydrofuran (5h) Yellow oil. ${ }^{9}$ IR (film): $\nu$ 3427, 3059, 3026, 2925, 1765, 1682, 1598, 1490, 1447, 1226, 1179, 1064, 758, $700 \mathrm{~cm}^{-1} .{ }^{1} \mathrm{H}$ NMR $\left(500 \mathrm{MHz}, \mathrm{CDCl}_{3}\right) \delta 4.11(\mathrm{q}, J=1 \mathrm{H} \mathrm{Hz}$, $7.1) 5.10(\mathrm{dd}, J=11.2,0,8 \mathrm{~Hz}, 1 \mathrm{H}) 5.31(\mathrm{dd}, J=17.7,0,8 \mathrm{~Hz}, 1 \mathrm{H}) 6.16-6.18(\mathrm{~m}, 1 \mathrm{H}) 6.20-6.27(\mathrm{~m}, 1 \mathrm{H})$ 7.10-7.40 (m, 10H) ppm. ${ }^{13} \mathrm{C}$ NMR $\left(125 \mathrm{MHz}, \mathrm{CDCl}_{3}\right) \delta \quad 60.394 .5,117.5,124.8,127.8,127.9,129.7$, 143.3, 143.6, 171.1, ppm. MS (EI) $\mathrm{m} / \mathrm{z}$ (rel intensity) $248\left(15\right.$, , $^{+\cdot}$ ) 229 (8) 215 (9) 205 (18) 204 (12) 203 (19) 191 (13) 189 (10) 183 (15) 182 (22) 172 (11) 171 (77) 165 (17) 157 (18) 143 (15) 141 (10) 129

\footnotetext{
${ }^{7}$ Nicolaou, K. C.; Hwang, C.-K.; Marron, B. E.; DeFrees, S. A.; Couladouros, E. A. J. Am. Chem. Soc. 1990, 112, 3040.

${ }^{8}$ Stille, J. K.; Becker, Y.J. Org. Chem. 1980, 45, 2139.

${ }^{9}$ Fürstner, A.; Ackermann, L.; Gabor, B.; Goddard, R.; Lehmann, C. W.; Mynott, R.; Stelzer, F. Thiel, O. R. Chem. Eur. J. $2001,7,3236$.

Supplementary data for $10.1021 / \mathrm{ja} 063186 \mathrm{w}$
} 
(10) 128 (22) 115 (23) 105 (100) 97 (14) 95 (9) 91 (34) 83 (11) 77 (43) 71 (16) 69 (15) 57 (20) 55 (14) 51 (14) 43 (41) 41 (12) 39 (9). HRMS (EI): calcd for [M] ${ }^{+\cdot}\left(\mathrm{C}_{18} \mathrm{H}_{16} \mathrm{O}\right)$ : 248.1201; found: 248.1196.

\section{(2E)-Octadec-2-en-1-yl acetate (5l)}

Colourless oil. ${ }^{10}$ Mixture of isomers $E: Z=88: 12$. IR (film): $\nu 2924,2854,1744,1464,1378,1363,1229$, $1024,967 \mathrm{~cm}^{-1}$. Isomer $E:{ }^{1} \mathrm{H} \mathrm{NMR}\left(400 \mathrm{MHz}, \mathrm{CDCl}_{3}\right) \delta 0.88(\mathrm{t}, J=6.7 \mathrm{~Hz}, 3 \mathrm{H}) 1.19-1.33(\mathrm{~m}, 26 \mathrm{H})$ 2.00-2.13 (m, 5H) $4.51(\mathrm{dd}, J=6.6,1,1 \mathrm{~Hz}, 2 \mathrm{H}) 5.48-5.60(\mathrm{~m}, 1 \mathrm{H}) 5.72-5.82(\mathrm{~m}, 1 \mathrm{H}) \mathrm{ppm}$. Isomer $E$ : ${ }^{13} \mathrm{C}$ NMR $\left(100 \mathrm{MHz}, \mathrm{CDCl}_{3}\right) \delta 14.1,21.0,22.7,26.9,28.9,29.2,29.3,29.4,29.5,29.6,29.7,30.1,32.0$, 32.2, 65.3, 135.5, 136.8, 170.9 ppm. MS (EI) $\mathrm{m} / z$ (rel intensity) $310\left(1,[\mathrm{M}]^{+\cdot}\right.$ ) 268 (9) 250 (10) 239 (5) 222 (11) 208 (4) 152 (3) 138 (4) 137 (4) 124 (7) 123 (8) 114 (10) 113 (5) 110 (14) 109 (13) 100 (8) 97 (20) 96 (49) 95 (25) 85 (5) 83 (24) 82 (48) 81 (31) 71 (10) 69 (24) 68 (35) 67 (29) 57 (25) 55 (42) 54 (28) 43 (100) 41 (40) 39 (6) Anal. calcd for $\mathrm{C}_{20} \mathrm{H}_{38} \mathrm{O}_{2}$ (310.5): C 77.36, H 12.33; found C 77.49, H 12.36. .

Metyl (2E)-4-(3-nitro-1,5-dioxaspiro[5.5] undec-3-yl)but-2-enoate (5j) White solid. IR (KBr): $\nu$ 2940, 2864, 1725, 1661, 1539, 1465, 1442, 1333, 1286, 1197, 1169, 1113, 1048, 1009, 988, 910, 855, 823, 764, 581, 549, $509 \mathrm{~cm}^{-1} .{ }^{1} \mathrm{H}$ NMR $\left(400 \mathrm{MHz}, \mathrm{CDCl}_{3}\right) \delta 1.38-1.46(\mathrm{~m}, 2 \mathrm{H}), 1.48-1.56(\mathrm{~m}, 4 \mathrm{H}), 1.66-1.72(\mathrm{~m}$, 2H), 1.72-1.80 (m, 2H), $2.79(\mathrm{dd}, J=7.8,1.4 \mathrm{~Hz}, 2 \mathrm{H}), 3.74(\mathrm{~s}, 3 \mathrm{H}), 3.98(\mathrm{~d}, J=12.8 \mathrm{~Hz}, 2 \mathrm{H}), 4.44(\mathrm{~d}$, $J=12.8 \mathrm{~Hz}, 2 \mathrm{H}), 5.94(\mathrm{dt}, J=15.4,1.4 \mathrm{~Hz}, 1 \mathrm{H}), 6.73(\mathrm{dt}, J=15.6,7.8 \mathrm{~Hz}, 1 \mathrm{H}) \mathrm{ppm} .{ }^{13} \mathrm{C}$ NMR $(100$ $\left.\mathrm{MHz}, \mathrm{CDCl}_{3}\right) \delta 22.3,22.5,25.3,31.0,33.0,36.4,51.8,62.8,85.2,99.4,126.7,138.1,165.6$. MS (EI) $\mathrm{m} / \mathrm{z}$ (rel intensity) 299 (25, $\mathrm{M}^{+\cdot}$ ), 270 (28), 256 (100), 154 (13), 123 (34), 97 (25), 95 (24), 79 (13), 69 (12), 67 (13), 55 (54), 41 (15). HRMS (EI) calcd for $\mathrm{M}^{+\cdot}\left(\mathrm{C}_{14} \mathrm{H}_{21} \mathrm{~N}_{1} \mathrm{O}_{6}\right)$ : 299.1369; found: 299.1363 


\section{Copies of ${ }^{1} \mathrm{H}$ and ${ }^{13} \mathrm{C}$-NMR spectra}
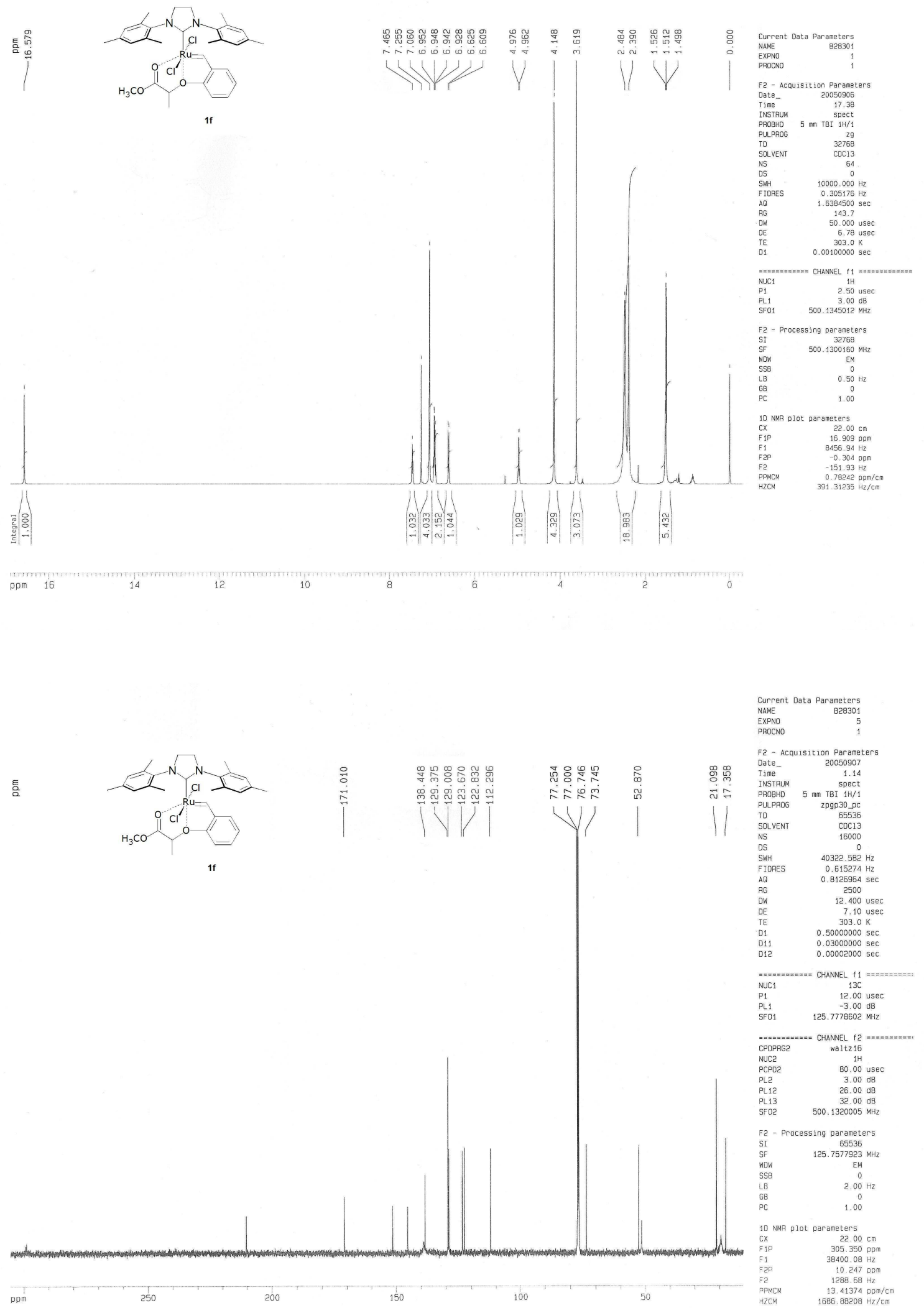

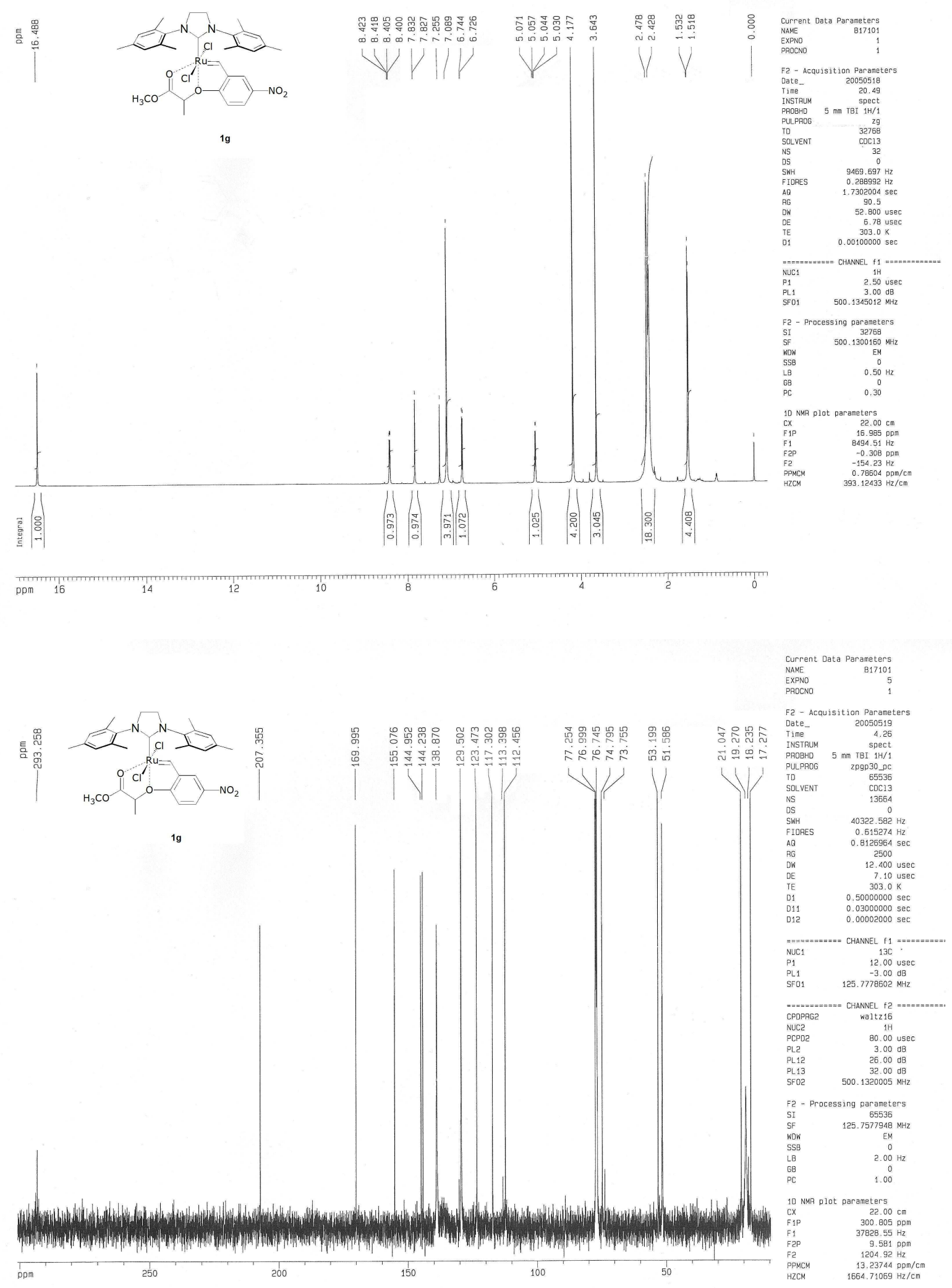


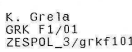

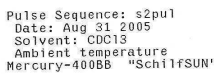

Relax. delay 1.000 se
Pulse 33.3 degrees

Acq time 4.736 s sc
width $4504.5 \mathrm{~Hz}$

OBERVE H1, 400.0418359 MHZ
OATA PROCESSING
FT SIZE 65536

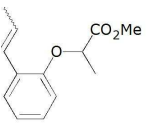

$3 \mathrm{f}$

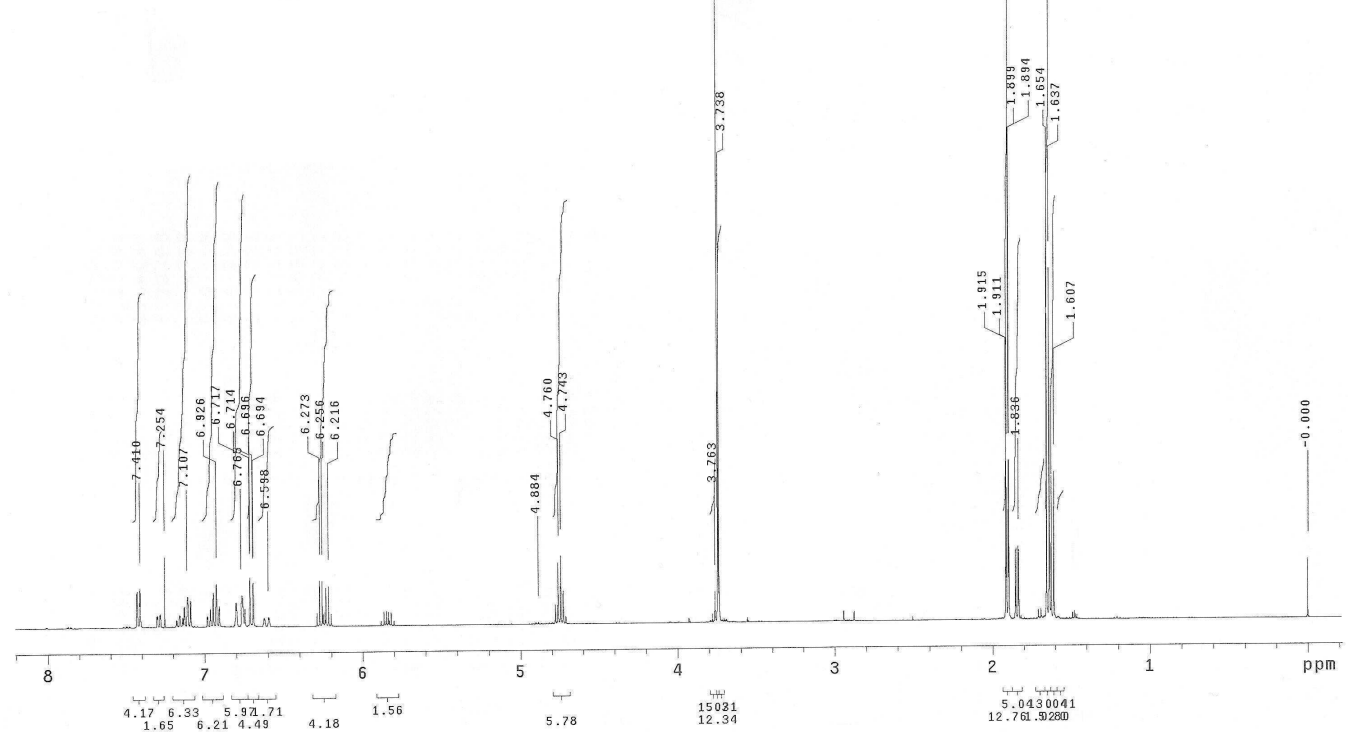

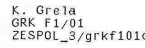

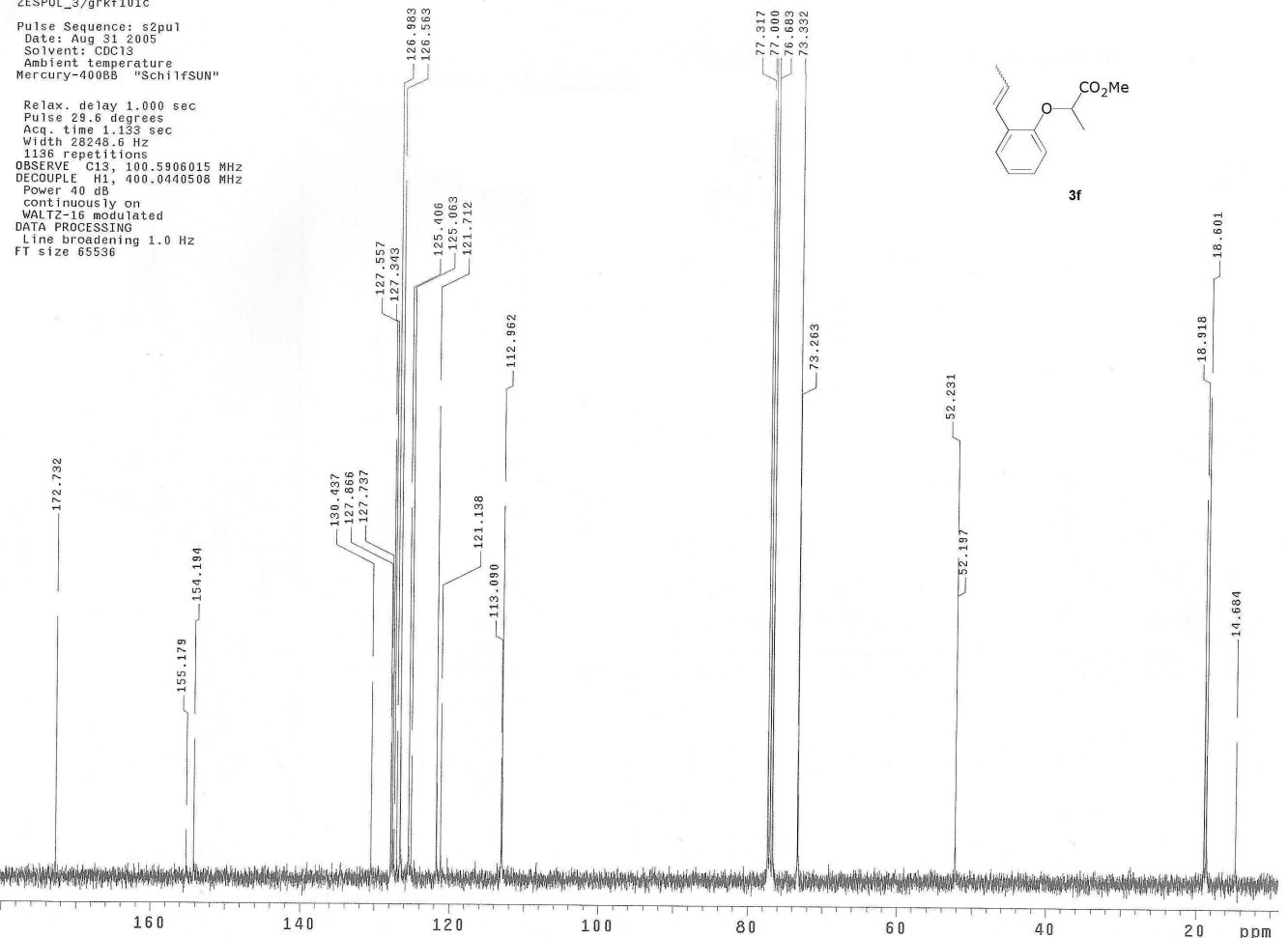




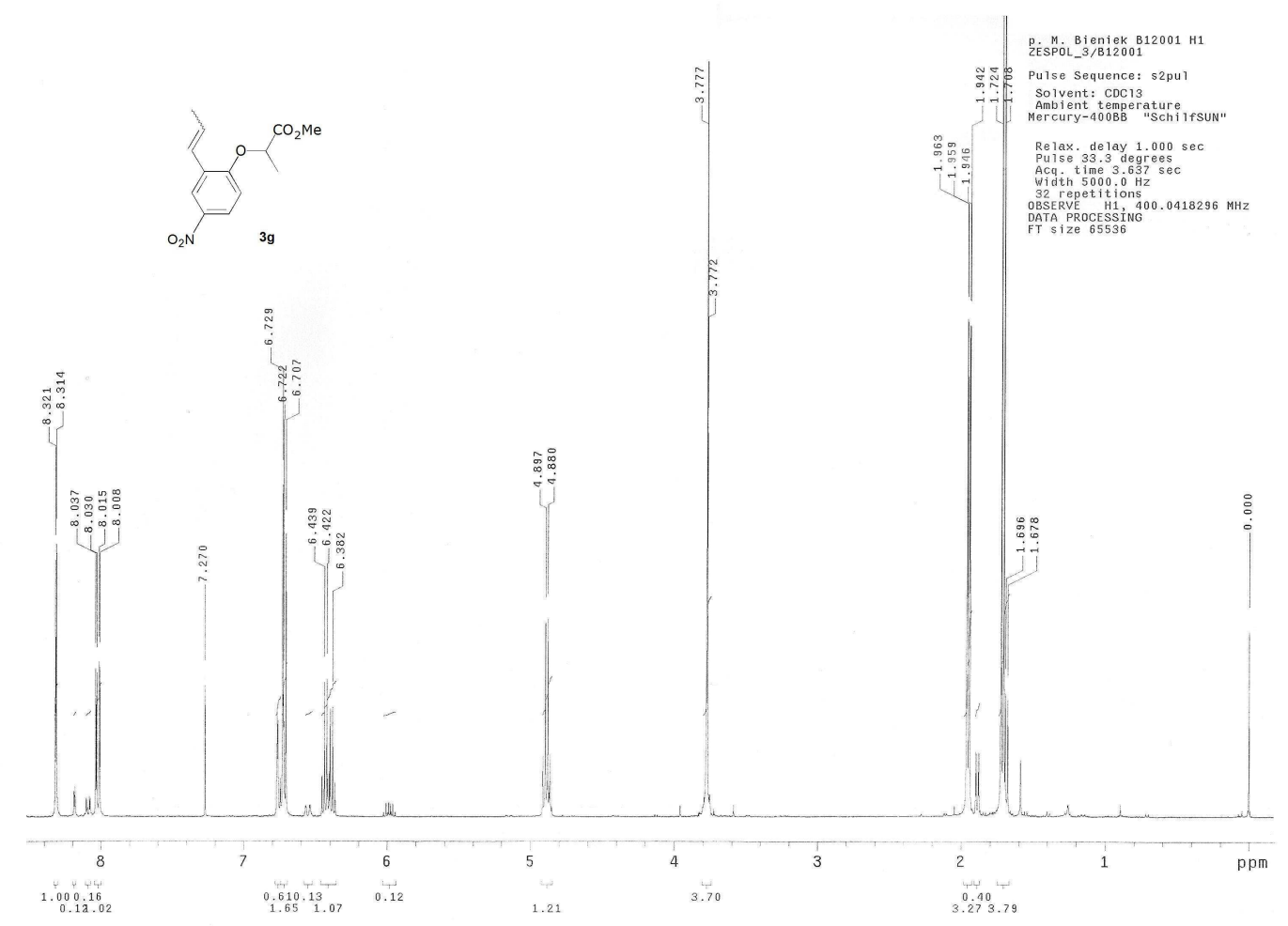

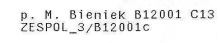

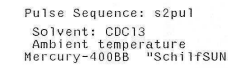

Relax. de lay $1.000 \mathrm{sec}$
Pulse 28.8 degres
Acr time
time 1 ige

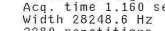

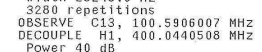

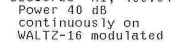

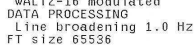

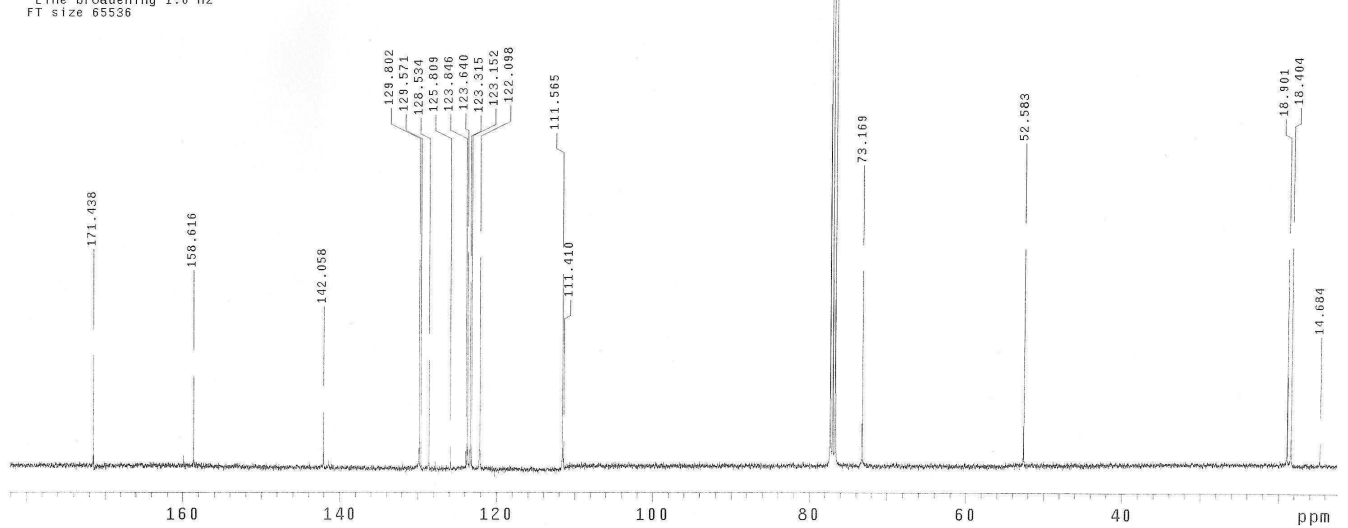




\section{X-ray diffraction}

\subsection{General}

Crystals of $\mathbf{1 f}$ suitable for X-ray diffraction were obtained through re-crystallization from ethyl acetate mixture at room temperature (1f was dissolved in minimal amount EtOAc and left in open flask overnight at room temperature). Intensities were collected at 180(2) $\mathrm{K}$ on an Oxford Diffraction Xcalibur diffractometer using graphite-monochromated $\operatorname{MoK}_{\alpha}$ radiation $(\lambda=0.71073 \AA$ A). Cell refinement and data processing were performed using Crysalis RED. ${ }^{11}$ All subsequent calculations were carried out using the WinGX system. ${ }^{12}$ Full crystallographic data are given in Table 1 . The structures were solved by using the SIR92 program, ${ }^{13}$ which revealed in each instance the position of most of the non-hydrogen atoms. All remaining non-hydrogen atoms were located by the usual combination of full matrix least-squares refinement and difference electron density syntheses by using the SHELXL97 program. ${ }^{14}$ The complex was found to crystallize with one molecule of (disordered) ethyl acetate per unit cell. All non-hydrogen atoms were refined with anisotropic displacement parameters. Hydrogen atoms were set in idealised positions and were treated as "riding" atoms with $\mathrm{C}\left(\mathrm{sp}^{2}\right)-\mathrm{H}=0.93 \AA^{\circ}$ or $\mathrm{C}\left(\mathrm{sp}^{3}\right)-\mathrm{H}=0.96 \stackrel{\circ}{\mathrm{A}}$, and $U_{i s o}(\mathrm{H})=1.2$ times that of the parent atom. Atomic scattering factors were taken from the usual tabulations. Anomalous dispersion terms for $\mathrm{Ru}, \mathrm{P}$, and $\mathrm{Cl}$ atoms were included in $F_{c c}$. Analytical ${ }^{15}$ absorption corrections were applied.

CCDC 282902 contains the supplementary crystallographic data for this paper. These data can be obtained free of charge via www.ccdc.cam.ac.uk/data_request/cif, or by emailing data_request@ccdc.cam.ac.uk, or by contacting The Cambridge Crystallographic Data Centre, 12, Union Road, Cambridge CB2 1EZ, UK; fax: +44 1223336033 .

\subsection{Molecular view of $1 \mathrm{f}$ in the solid state}

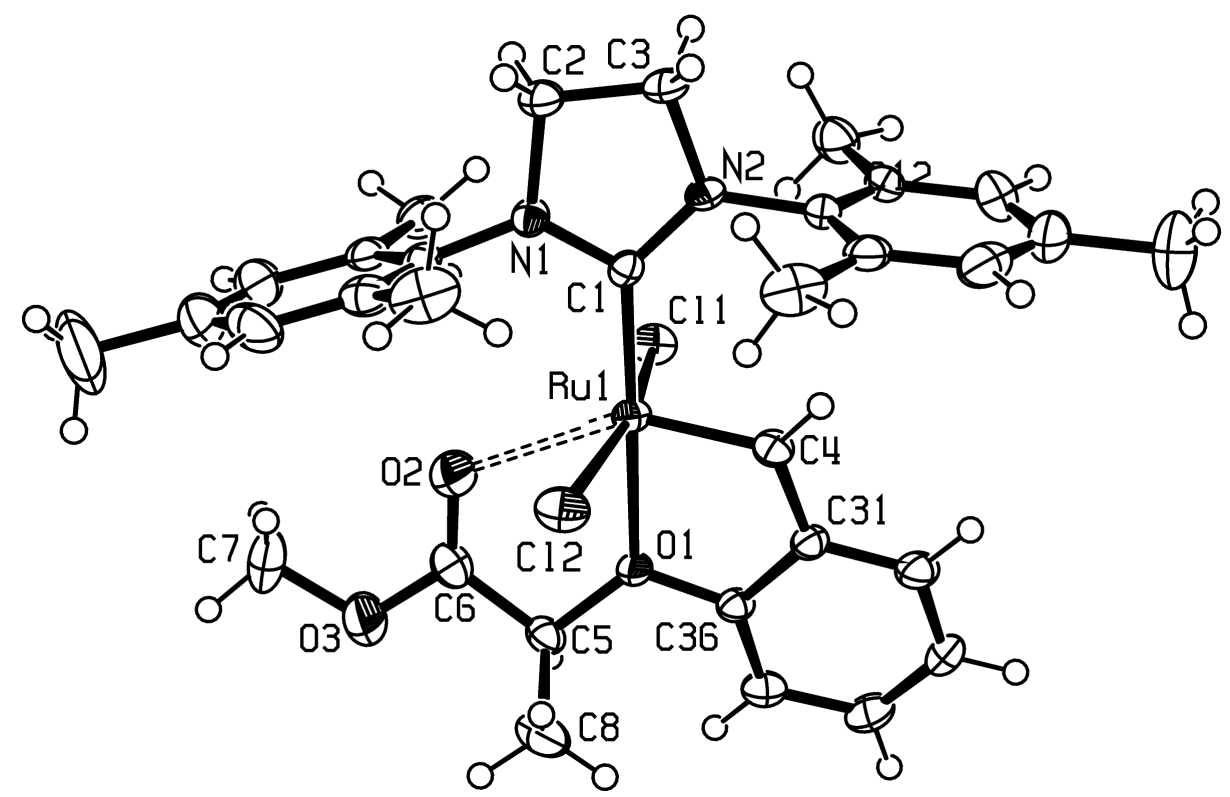

Figure 1. Molecular view of $\mathbf{1 f}$ in the solid state. Selected bond lengths $[\AA]$ and angles $\left[^{\circ}\right]$ : $\mathrm{O}(1)-\operatorname{Ru}(1) 2.207, \mathrm{O}(2)-\mathrm{Ru}(1) 2.536$, $\mathrm{C}(1)-\mathrm{Ru}(1)$ 1.984, C(4)-Ru(1) 1.820, Cl(1)-Ru(1) 2.3583, Cl(2)-Ru(1) 2.3560, C(1)-Ru(1)-O(1) 176.56, C(4)-Ru(1)-O(2) 146.92, $\mathrm{Cl}(2)-\mathrm{Ru}(1)-\mathrm{Cl}(1) 164.63$.

\footnotetext{
${ }^{11}$ CrysAlis RED (Version 1.170.32), Oxford Diffraction Ltd., Oxford, United Kingdom, 2003.

12 L. J. Farrugia, J. Appl. Crystallogr. 1999, 32, 837-838.

13 A. Altomare, G. Cascarano, C. Giacovazzo, A. Guagliardi, J. Appl. Crystallogr. 1993, 26, 343-350

${ }^{14}$ SHELX97 (Includes SHELXS97, SHELXL97, CIFTAB) - Programs for Crystal Structure Analysis (Release 97-2). G. M. Sheldrick, Institüt für Anorganische Chemie der Universität, Tammanstrasse 4, D-3400 Göttingen, Germany, 1998.

15 N. W. Alcock, 1970, The Analytical Method for Absorption Correction, Crystallographic Computing, F.R Ahmed, S.R. Hall, and C.P. Huber, eds., Munksgaard, Copenhagen, pp. 271-278.

Supplementary data for $10.1021 / \mathrm{ja} 063186 \mathrm{w}$ 


\subsection{Tabular data}

Table 1. Crystal data and structure refinement for mb041004.

Identification code mb041004

Empirical formula C36 $\mathrm{H} 46 \mathrm{Cl} 2 \mathrm{~N} 2 \mathrm{O} 5 \mathrm{Ru}$

Formula weight 758.72

Temperature 180(2) $\mathrm{K}$

Wavelength $0.71073 \stackrel{\circ}{A}$

Crystal system monoclinic

Space group P 21/c

Unit cell dimensions $\mathrm{a}=13.6327(18){ }^{\circ} \mathrm{A} \alpha=90^{\circ}$.

$\mathrm{b}=10.4005(13) \AA \mathrm{A} \beta=101.221(11)^{\circ}$.

$\mathrm{c}=26.417(3) \stackrel{\circ}{\mathrm{A}} \gamma=90^{\circ}$

Volume 3674.0(8) $\stackrel{\circ}{ }^{3}$

Z 4

Density (calculated) $1.372 \mathrm{Mg} / \mathrm{m}^{3}$

Absorption coefficient $0.614 \mathrm{~mm}^{-1}$

$\mathrm{F}(000) 1576$

Crystal size 0.34 x $0.164 \times 0.0 .078 \mathrm{~mm}^{3}$

Theta range for data collection 3.09 to $32.21^{\circ}$.

Index ranges $-20<=\mathrm{h}<=18,-15<=\mathrm{k}<=14,-39<=\mathrm{l}<=37$

Reflections collected 37120

Independent reflections $12202[\mathrm{R}(\mathrm{int})=0.1065]$

Completeness to theta $=32.21^{\circ} 93.9 \%$

Absorption correction Analytical (faces)

Max. and min. transmission 0.9590 and 0.7805

Refinement method Full-matrix least-squares on $\mathrm{F}^{2}$

Data / restraints / parameters 12202 / 0 / 435

Goodness-of-fit on $\mathrm{F}^{2} 0.820$

Final $R$ indices $[\mathrm{I} \leq 2 \operatorname{sigma}(\mathrm{I})] \mathrm{R} 1=0.0544, \mathrm{wR} 2=0.0806$

$\mathrm{R}$ indices (all data) $\mathrm{R} 1=0.1550, \mathrm{wR} 2=0.1019$

Largest diff. peak and hole 0.669 and -0.669 e..$^{-3}$

Table 2. Atomic coordinates $\left(\mathrm{x} 10^{\wedge} 4\right)$ and equivalent isotropic displacement parameters $\left(A^{\wedge} 2 \times 10^{\wedge} 3\right)$ for mb041004. $U($ eq) is defined as one third of the trace of the orthogonalized $U^{\wedge}(i j)$ tensor.

$\begin{array}{lll}\mathrm{x} & \mathrm{y} & \mathrm{z} \mathrm{U}(\mathrm{eq})\end{array}$




\begin{tabular}{|c|c|c|c|c|}
\hline$C(1)$ & $9269(3)$ & $3528(3)$ & $3381(1)$ & $19(1)$ \\
\hline$C(2)$ & $9535(3)$ & $5709(3)$ & $3175(2)$ & $28(1)$ \\
\hline$C(3)$ & $8459(3)$ & $5349(3)$ & $2957(2)$ & $28(1)$ \\
\hline$C(4)$ & $8494(3)$ & $932(3)$ & $3472(1)$ & $24(1)$ \\
\hline$C(5)$ & $10936(3)$ & $-307(3)$ & $4461(1)$ & $25(1)$ \\
\hline$C(6)$ & $11554(3)$ & $897(4)$ & $4515(1)$ & $29(1)$ \\
\hline$C(7)$ & $13179(3)$ & $1722(5)$ & $4787(2)$ & $62(2)$ \\
\hline$C(8)$ & $11349(3)$ & $-1377(4)$ & $4162(2)$ & $41(1)$ \\
\hline$C(11)$ & $7464(3)$ & $3360(3)$ & $2939(1)$ & $24(1)$ \\
\hline$C(12)$ & $6689(3)$ & $3444(3)$ & $3197(2)$ & $29(1)$ \\
\hline$C(13)$ & $5790(3)$ & $2844(4)$ & $2976(2)$ & $43(1)$ \\
\hline$C(14)$ & $5676(3)$ & $2178(4)$ & $2514(2)$ & $48(1)$ \\
\hline$C(15)$ & $6477(3)$ & $2126(4)$ & $2272(2)$ & $41(1)$ \\
\hline$C(16)$ & $7391(3)$ & $2711(4)$ & $2473(2)$ & $30(1)$ \\
\hline$C(17)$ & $6776(3)$ & $4179(4)$ & $3695(2)$ & $37(1)$ \\
\hline$C(18)$ & $4691(3)$ & $1531(5)$ & $2286(2)$ & $80(2)$ \\
\hline C (19) & $8267(3)$ & $2593(4)$ & $2197(2)$ & $43(1)$ \\
\hline$C(21)$ & $11012(3)$ & $4427(3)$ & 3591 (1) & $24(1)$ \\
\hline$C(22)$ & $11666(3)$ & $4219(4)$ & $3251(2)$ & $32(1)$ \\
\hline$C(23)$ & $12683(3)$ & $4311(4)$ & $3445(2)$ & $46(1)$ \\
\hline$C(24)$ & $13059(3)$ & $4660(5)$ & $3943(2)$ & $50(1)$ \\
\hline$C(25)$ & $12409(3)$ & $4895(4)$ & $4275(2)$ & $41(1)$ \\
\hline$C(26)$ & $11369(3)$ & $4783(3)$ & $4101(2)$ & $26(1)$ \\
\hline$C(27)$ & $11278(3)$ & $3945(4)$ & $2683(2)$ & $47(1)$ \\
\hline$C(28)$ & $14188(3)$ & $4788(6)$ & $4134(2)$ & $100(2)$ \\
\hline$C(29)$ & $10684(3)$ & $5047(4)$ & $4473(1)$ & $32(1)$ \\
\hline$C(31)$ & $8389(3)$ & $-304(3)$ & $3715(1)$ & $22(1)$ \\
\hline$C(32)$ & $7529(3)$ & $-1068(4)$ & $3593(2)$ & $31(1)$ \\
\hline$C(33)$ & $7454(3)$ & $-2208(4)$ & $3851(2)$ & $35(1)$ \\
\hline$C(34)$ & $8229(3)$ & $-2614(4)$ & $4232(2)$ & $35(1)$ \\
\hline$C(35)$ & $9106(3)$ & $-1885(4)$ & $4363(1)$ & $28(1)$ \\
\hline$C(36)$ & $9166(3)$ & $-748(3)$ & $4104(1)$ & $21(1)$ \\
\hline$C(96)$ & $13828(4)$ & $-1901(6)$ & $5280(2)$ & $88(2)$ \\
\hline C (97) & $13889(4)$ & $-1108(5)$ & $5752(2)$ & $78(2)$ \\
\hline$C(98)$ & $15382(6)$ & $-98(9)$ & $6166(4)$ & $127(4)$ \\
\hline O(99) & $16463(4)$ & $-283(7)$ & $6481(2)$ & $188(3)$ \\
\hline $\mathrm{C}(99)$ & $14865(8)$ & $525(13)$ & $6435(6)$ & $96(5)$ \\
\hline$C\left(99^{\prime}\right)$ & $15103(10)$ & $826(13)$ & $5839(6)$ & $99(5)$ \\
\hline$N(1)$ & $9953(2)$ & $4470(3)$ & $3388(1)$ & $22(1)$ \\
\hline$N(2)$ & $8389(2)$ & $4031(3)$ & $3140(1)$ & $23(1)$ \\
\hline$O(1)$ & $9954(2)$ & $94(2)$ & $4202(1)$ & $26(1)$ \\
\hline$O(2)$ & $11258(2)$ & $1943(3)$ & $4375(1)$ & $39(1)$ \\
\hline $0(3)$ & $12488(2)$ & $640(3)$ & $4737(1)$ & $51(1)$ \\
\hline $0(98)$ & $14958(3)$ & $-1105(5)$ & $6012(2)$ & $108(2)$ \\
\hline $\mathrm{Cl}(1)$ & $8933(1)$ & $2602(1)$ & $4459(1)$ & $28(1)$ \\
\hline $\mathrm{Cl}(2)$ & 10654 (1) & $1139(1)$ & $3205(1)$ & $33(1)$ \\
\hline $\mathrm{Ru}(1)$ & $9593(1)$ & $1873(1)$ & $3749(1)$ & $21(1)$ \\
\hline
\end{tabular}

Table 3. Bond lengths [A] and angles [o] for mb041004. 


\begin{tabular}{|c|c|}
\hline$C(1)-N(2)$ & $1.349(4)$ \\
\hline$C(1)-N(1)$ & $1.351(4)$ \\
\hline$C(1)-R u(1)$ & $1.984(3)$ \\
\hline $\mathrm{C}(2)-\mathrm{N}(1)$ & $1.475(4)$ \\
\hline$C(2)-C(3)$ & $1.516(5)$ \\
\hline $\mathrm{C}(2)-\mathrm{H}(2 \mathrm{~A})$ & 0.9700 \\
\hline $\mathrm{C}(2)-\mathrm{H}(2 \mathrm{~B})$ & 0.9700 \\
\hline $\mathrm{C}(3)-\mathrm{N}(2)$ & $1.463(4)$ \\
\hline$C(3)-H(3 A)$ & 0.9700 \\
\hline$C(3)-H(3 B)$ & 0.9700 \\
\hline$C(4)-C(31)$ & $1.456(5)$ \\
\hline$C(4)-R u(1)$ & $1.820(3)$ \\
\hline $\mathrm{C}(4)-\mathrm{H}(4)$ & 0.9300 \\
\hline$C(5)-0(1)$ & $1.442(4)$ \\
\hline$C(5)-C(6)$ & $1.500(5)$ \\
\hline$C(5)-C(8)$ & $1.534(5)$ \\
\hline $\mathrm{C}(5)-\mathrm{H}(5)$ & 0.9800 \\
\hline$C(6)-0(2)$ & $1.194(4)$ \\
\hline$C(6)-0(3)$ & $1.321(4)$ \\
\hline$C(7)-0(3)$ & $1.457(5)$ \\
\hline $\mathrm{C}(7)-\mathrm{H}(7 \mathrm{~A})$ & 0.9600 \\
\hline$C(7)-H(7 B)$ & 0.9600 \\
\hline $\mathrm{C}(7)-\mathrm{H}(7 \mathrm{C})$ & 0.9600 \\
\hline $\mathrm{C}(8)-\mathrm{H}(8 \mathrm{~A})$ & 0.9600 \\
\hline $\mathrm{C}(8)-\mathrm{H}(8 \mathrm{~B})$ & 0.9600 \\
\hline$C(8)-H(8 C)$ & 0.9600 \\
\hline$C(11)-C(12)$ & $1.366(5)$ \\
\hline$C(11)-C(16)$ & $1.390(5)$ \\
\hline $\mathrm{C}(11)-\mathrm{N}(2)$ & $1.449(4)$ \\
\hline$C(12)-C(13)$ & $1.397(5)$ \\
\hline$C(12)-C(17)$ & $1.506(5)$ \\
\hline$C(13)-C(14)$ & $1.385(6)$ \\
\hline $\mathrm{C}(13)-\mathrm{H}(13)$ & 0.9300 \\
\hline$C(14)-C(15)$ & $1.370(6)$ \\
\hline$C(14)-C(18)$ & $1.518(5)$ \\
\hline$C(15)-C(16)$ & $1.396(5)$ \\
\hline $\mathrm{C}(15)-\mathrm{H}(15)$ & 0.9300 \\
\hline$C(16)-C(19)$ & $1.522(5)$ \\
\hline $\mathrm{C}(17)-\mathrm{H}(17 \mathrm{~A})$ & 0.9600 \\
\hline $\mathrm{C}(17)-\mathrm{H}(17 \mathrm{~B})$ & 0.9600 \\
\hline $\mathrm{C}(17)-\mathrm{H}(17 \mathrm{C})$ & 0.9600 \\
\hline $\mathrm{C}(18)-\mathrm{H}(18 \mathrm{~A})$ & 0.9600 \\
\hline $\mathrm{C}(18)-\mathrm{H}(18 \mathrm{~B})$ & 0.9600 \\
\hline $\mathrm{C}(18)-\mathrm{H}(18 \mathrm{C})$ & 0.9600 \\
\hline$C(19)-H(19 A)$ & 0.9600 \\
\hline$C(19)-H(19 B)$ & 0.9600 \\
\hline $\mathrm{C}(19)-\mathrm{H}(19 \mathrm{C})$ & 0.9600 \\
\hline$C(21)-C(26)$ & $1.390(5)$ \\
\hline$C(21)-C(22)$ & $1.399(5)$ \\
\hline $\mathrm{C}(21)-\mathrm{N}(1)$ & $1.439(4)$ \\
\hline
\end{tabular}

Supplementary data for $10.1021 / \mathrm{ja} 063186 \mathrm{w}$ 


\begin{tabular}{|c|c|}
\hline$C(22)-C(23)$ & $1.386(5)$ \\
\hline$C(22)-C(27)$ & $1.518(5)$ \\
\hline$C(23)-C(24)$ & $1.366(6)$ \\
\hline $\mathrm{C}(23)-\mathrm{H}(23)$ & 0.9300 \\
\hline$C(24)-C(25)$ & $1.383(6)$ \\
\hline$C(24)-C(28)$ & $1.529(6)$ \\
\hline$C(25)-C(26)$ & $1.408(5)$ \\
\hline $\mathrm{C}(25)-\mathrm{H}(25)$ & 0.9300 \\
\hline$C(26)-C(29)$ & $1.507(5)$ \\
\hline $\mathrm{C}(27)-\mathrm{H}(27 \mathrm{~A})$ & 0.9600 \\
\hline $\mathrm{C}(27)-\mathrm{H}(27 \mathrm{~B})$ & 0.9600 \\
\hline $\mathrm{C}(27)-\mathrm{H}(27 \mathrm{C})$ & 0.9600 \\
\hline $\mathrm{C}(28)-\mathrm{H}(28 \mathrm{~A})$ & 0.9600 \\
\hline $\mathrm{C}(28)-\mathrm{H}(28 \mathrm{~B})$ & 0.9600 \\
\hline $\mathrm{C}(28)-\mathrm{H}(28 \mathrm{C})$ & 0.9600 \\
\hline $\mathrm{C}(29)-\mathrm{H}(29 \mathrm{~A})$ & 0.9600 \\
\hline $\mathrm{C}(29)-\mathrm{H}(29 \mathrm{~B})$ & 0.9600 \\
\hline $\mathrm{C}(29)-\mathrm{H}(29 \mathrm{C})$ & 0.9600 \\
\hline$C(31)-C(32)$ & $1.400(5)$ \\
\hline$C(31)-C(36)$ & $1.403(4)$ \\
\hline$C(32)-C(33)$ & $1.380(5)$ \\
\hline $\mathrm{C}(32)-\mathrm{H}(32)$ & 0.9300 \\
\hline$C(33)-C(34)$ & $1.377(5)$ \\
\hline $\mathrm{C}(33)-\mathrm{H}(33)$ & 0.9300 \\
\hline$C(34)-C(35)$ & $1.401(5)$ \\
\hline $\mathrm{C}(34)-\mathrm{H}(34)$ & 0.9300 \\
\hline$C(35)-C(36)$ & $1.377(5)$ \\
\hline $\mathrm{C}(35)-\mathrm{H}(35)$ & 0.9300 \\
\hline$C(36)-0(1)$ & $1.371(4)$ \\
\hline$C(96)-C(97)$ & $1.484(7)$ \\
\hline $\mathrm{C}(96)-\mathrm{H}(96 \mathrm{~A})$ & 0.9600 \\
\hline $\mathrm{C}(96)-\mathrm{H}(96 \mathrm{~B})$ & 0.9600 \\
\hline $\mathrm{C}(96)-\mathrm{H}(96 \mathrm{C})$ & 0.9600 \\
\hline$C(97)-0(98)$ & $1.486(6)$ \\
\hline $\mathrm{C}(97)-\mathrm{H}(97 \mathrm{~A})$ & 0.9700 \\
\hline $\mathrm{C}(97)-\mathrm{H}(97 \mathrm{~B})$ & 0.9700 \\
\hline$C(98)-0(98)$ & $1.227(7)$ \\
\hline$C(98)-C(99)$ & $1.272(12)$ \\
\hline $\mathrm{C}(98)-\mathrm{C}\left(99^{\prime}\right)$ & $1.300(12)$ \\
\hline$C(98)-0(99)$ & $1.557(8)$ \\
\hline $\mathrm{C}(99)-\mathrm{H}(99 \mathrm{~A})$ & 0.9600 \\
\hline $\mathrm{C}(99)-\mathrm{H}(99 \mathrm{~B})$ & 0.9600 \\
\hline $\mathrm{C}(99)-\mathrm{H}(99 \mathrm{C})$ & 0.9600 \\
\hline $\mathrm{C}\left(99^{\prime}\right)-\mathrm{H}(99 \mathrm{D})$ & 0.9600 \\
\hline $\mathrm{C}\left(99^{\prime}\right)-\mathrm{H}(99 \mathrm{E})$ & 0.9600 \\
\hline $\mathrm{C}\left(99^{\prime}\right)-\mathrm{H}(99 \mathrm{~F})$ & 0.9600 \\
\hline$O(1)-R u(1)$ & $2.207(2)$ \\
\hline $\mathrm{Cl}(1)-\mathrm{Ru}(1)$ & $2.3583(10)$ \\
\hline $\mathrm{Cl}(2)-\mathrm{Ru}(1)$ & $2.3560(11)$ \\
\hline$N(2)-C(1)-N(1)$ & $105.9(3)$ \\
\hline
\end{tabular}

Supplementary data for $10.1021 / \mathrm{ja} 063186 \mathrm{w}$ 


\begin{tabular}{|c|c|}
\hline$N(2)-C(1)-R u(1)$ & $131.4(3)$ \\
\hline$N(1)-C(1)-R u(1)$ & $122.3(2)$ \\
\hline$N(1)-C(2)-C(3)$ & $101.7(3)$ \\
\hline$N(1)-C(2)-H(2 A)$ & 111.4 \\
\hline$C(3)-C(2)-H(2 A)$ & 111.4 \\
\hline$N(1)-C(2)-H(2 B)$ & 111.4 \\
\hline$C(3)-C(2)-H(2 B)$ & 111.4 \\
\hline $\mathrm{H}(2 \mathrm{~A})-\mathrm{C}(2)-\mathrm{H}(2 \mathrm{~B})$ & 109.3 \\
\hline$N(2)-C(3)-C(2)$ & $103.0(3)$ \\
\hline$N(2)-C(3)-H(3 A)$ & 111.2 \\
\hline$C(2)-C(3)-H(3 A)$ & 111.2 \\
\hline$N(2)-C(3)-H(3 B)$ & 111.2 \\
\hline$C(2)-C(3)-H(3 B)$ & 111.2 \\
\hline $\mathrm{H}(3 \mathrm{~A})-\mathrm{C}(3)-\mathrm{H}(3 \mathrm{~B})$ & 109.1 \\
\hline$C(31)-C(4)-R u(1)$ & $116.1(2)$ \\
\hline $\mathrm{C}(31)-\mathrm{C}(4)-\mathrm{H}(4)$ & 121.9 \\
\hline $\mathrm{Ru}(1)-\mathrm{C}(4)-\mathrm{H}(4)$ & 121.9 \\
\hline$O(1)-C(5)-C(6)$ & $104.8(3)$ \\
\hline$O(1)-C(5)-C(8)$ & $111.5(3)$ \\
\hline$C(6)-C(5)-C(8)$ & $113.6(3)$ \\
\hline $\mathrm{O}(1)-\mathrm{C}(5)-\mathrm{H}(5)$ & 108.9 \\
\hline $\mathrm{C}(6)-\mathrm{C}(5)-\mathrm{H}(5)$ & 108.9 \\
\hline $\mathrm{C}(8)-\mathrm{C}(5)-\mathrm{H}(5)$ & 108.9 \\
\hline$O(2)-C(6)-0(3)$ & $124.1(4)$ \\
\hline$O(2)-C(6)-C(5)$ & $125.6(3)$ \\
\hline$O(3)-C(6)-C(5)$ & $110.3(3)$ \\
\hline $\mathrm{O}(3)-\mathrm{C}(7)-\mathrm{H}(7 \mathrm{~A})$ & 109.5 \\
\hline $\mathrm{O}(3)-\mathrm{C}(7)-\mathrm{H}(7 \mathrm{~B})$ & 109.5 \\
\hline $\mathrm{H}(7 \mathrm{~A})-\mathrm{C}(7)-\mathrm{H}(7 \mathrm{~B})$ & 109.5 \\
\hline $\mathrm{O}(3)-\mathrm{C}(7)-\mathrm{H}(7 \mathrm{C})$ & 109.5 \\
\hline $\mathrm{H}(7 \mathrm{~A})-\mathrm{C}(7)-\mathrm{H}(7 \mathrm{C})$ & 109.5 \\
\hline $\mathrm{H}(7 \mathrm{~B})-\mathrm{C}(7)-\mathrm{H}(7 \mathrm{C})$ & 109.5 \\
\hline$C(5)-C(8)-H(8 A)$ & 109.5 \\
\hline$C(5)-C(8)-H(8 B)$ & 109.5 \\
\hline $\mathrm{H}(8 \mathrm{~A})-\mathrm{C}(8)-\mathrm{H}(8 \mathrm{~B})$ & 109.5 \\
\hline$C(5)-C(8)-H(8 C)$ & 109.5 \\
\hline $\mathrm{H}(8 \mathrm{~A})-\mathrm{C}(8)-\mathrm{H}(8 \mathrm{C})$ & 109.5 \\
\hline $\mathrm{H}(8 \mathrm{~B})-\mathrm{C}(8)-\mathrm{H}(8 \mathrm{C})$ & 109.5 \\
\hline$C(12)-C(11)-C(16)$ & $122.9(3)$ \\
\hline$C(12)-C(11)-N(2)$ & $119.3(3)$ \\
\hline$C(16)-C(11)-N(2)$ & $117.7(3)$ \\
\hline$C(11)-C(12)-C(13)$ & $117.6(4)$ \\
\hline$C(11)-C(12)-C(17)$ & $122.0(3)$ \\
\hline$C(13)-C(12)-C(17)$ & $120.3(4)$ \\
\hline$C(14)-C(13)-C(12)$ & $122.0(4)$ \\
\hline$C(14)-C(13)-H(13)$ & 119.0 \\
\hline$C(12)-C(13)-H(13)$ & 119.0 \\
\hline$C(15)-C(14)-C(13)$ & $117.9(4)$ \\
\hline$C(15)-C(14)-C(18)$ & $121.4(5)$ \\
\hline$C(13)-C(14)-C(18)$ & $120.6(5)$ \\
\hline$C(14)-C(15)-C(16)$ & $122.6(4)$ \\
\hline
\end{tabular}

Supplementary data for $10.1021 / \mathrm{ja} 063186 \mathrm{w}$ 


\begin{tabular}{|c|c|}
\hline$C(14)-C(15)-H(15)$ & 118.7 \\
\hline$C(16)-C(15)-H(15)$ & 118.7 \\
\hline$C(11)-C(16)-C(15)$ & $117.0(4)$ \\
\hline$C(11)-C(16)-C(19)$ & $122.5(3)$ \\
\hline$C(15)-C(16)-C(19)$ & $120.5(4)$ \\
\hline$C(12)-C(17)-H(17 A)$ & 109.5 \\
\hline $\mathrm{C}(12)-\mathrm{C}(17)-\mathrm{H}(17 \mathrm{~B})$ & 109.5 \\
\hline$H(17 A)-C(17)-H(17 B)$ & 109.5 \\
\hline $\mathrm{C}(12)-\mathrm{C}(17)-\mathrm{H}(17 \mathrm{C})$ & 109.5 \\
\hline $\mathrm{H}(17 \mathrm{~A})-\mathrm{C}(17)-\mathrm{H}(17 \mathrm{C})$ & 109.5 \\
\hline$H(17 B)-C(17)-H(17 C)$ & 109.5 \\
\hline$C(14)-C(18)-H(18 A)$ & 109.5 \\
\hline $\mathrm{C}(14)-\mathrm{C}(18)-\mathrm{H}(18 \mathrm{~B})$ & 109.5 \\
\hline $\mathrm{H}(18 \mathrm{~A})-\mathrm{C}(18)-\mathrm{H}(18 \mathrm{~B})$ & 109.5 \\
\hline$C(14)-C(18)-H(18 C)$ & 109.5 \\
\hline $\mathrm{H}(18 \mathrm{~A})-\mathrm{C}(18)-\mathrm{H}(18 \mathrm{C})$ & 109.5 \\
\hline $\mathrm{H}(18 \mathrm{~B})-\mathrm{C}(18)-\mathrm{H}(18 \mathrm{C})$ & 109.5 \\
\hline$C(16)-C(19)-H(19 A)$ & 109.5 \\
\hline$C(16)-C(19)-H(19 B)$ & 109.5 \\
\hline$H(19 A)-C(19)-H(19 B)$ & 109.5 \\
\hline$C(16)-C(19)-H(19 C)$ & 109.5 \\
\hline$H(19 A)-C(19)-H(19 C)$ & 109.5 \\
\hline$H(19 B)-C(19)-H(19 C)$ & 109.5 \\
\hline$C(26)-C(21)-C(22)$ & $121.2(4)$ \\
\hline$C(26)-C(21)-N(1)$ & $119.2(3)$ \\
\hline$C(22)-C(21)-N(1)$ & $118.9(3)$ \\
\hline$C(23)-C(22)-C(21)$ & $117.9(4)$ \\
\hline$C(23)-C(22)-C(27)$ & $120.7(4)$ \\
\hline$C(21)-C(22)-C(27)$ & $121.4(4)$ \\
\hline$C(24)-C(23)-C(22)$ & $122.4(4)$ \\
\hline$C(24)-C(23)-H(23)$ & 118.8 \\
\hline$C(22)-C(23)-H(23)$ & 118.8 \\
\hline$C(23)-C(24)-C(25)$ & $119.4(4)$ \\
\hline$C(23)-C(24)-C(28)$ & $120.3(5)$ \\
\hline$C(25)-C(24)-C(28)$ & $120.3(5)$ \\
\hline$C(24)-C(25)-C(26)$ & $120.5(4)$ \\
\hline$C(24)-C(25)-H(25)$ & 119.8 \\
\hline$C(26)-C(25)-H(25)$ & 119.8 \\
\hline$C(21)-C(26)-C(25)$ & $118.6(4)$ \\
\hline$C(21)-C(26)-C(29)$ & $122.5(3)$ \\
\hline$C(25)-C(26)-C(29)$ & $118.9(4)$ \\
\hline $\mathrm{C}(22)-\mathrm{C}(27)-\mathrm{H}(27 \mathrm{~A})$ & 109.5 \\
\hline $\mathrm{C}(22)-\mathrm{C}(27)-\mathrm{H}(27 \mathrm{~B})$ & 109.5 \\
\hline $\mathrm{H}(27 \mathrm{~A})-\mathrm{C}(27)-\mathrm{H}(27 \mathrm{~B})$ & 109.5 \\
\hline $\mathrm{C}(22)-\mathrm{C}(27)-\mathrm{H}(27 \mathrm{C})$ & 109.5 \\
\hline $\mathrm{H}(27 \mathrm{~A})-\mathrm{C}(27)-\mathrm{H}(27 \mathrm{C})$ & 109.5 \\
\hline$H(27 B)-C(27)-H(27 C)$ & 109.5 \\
\hline $\mathrm{C}(24)-\mathrm{C}(28)-\mathrm{H}(28 \mathrm{~A})$ & 109.5 \\
\hline $\mathrm{C}(24)-\mathrm{C}(28)-\mathrm{H}(28 \mathrm{~B})$ & 109.5 \\
\hline $\mathrm{H}(28 \mathrm{~A})-\mathrm{C}(28)-\mathrm{H}(28 \mathrm{~B})$ & 109.5 \\
\hline $\mathrm{C}(24)-\mathrm{C}(28)-\mathrm{H}(28 \mathrm{C})$ & 109.5 \\
\hline
\end{tabular}

Supplementary data for $10.1021 / \mathrm{ja} 063186 \mathrm{w}$ 


\begin{tabular}{|c|c|}
\hline $\mathrm{H}(28 \mathrm{~A})-\mathrm{C}(28)-\mathrm{H}(28 \mathrm{C})$ & 109.5 \\
\hline $\mathrm{H}(28 \mathrm{~B})-\mathrm{C}(28)-\mathrm{H}(28 \mathrm{C})$ & 109.5 \\
\hline $\mathrm{C}(26)-\mathrm{C}(29)-\mathrm{H}(29 \mathrm{~A})$ & 109.5 \\
\hline $\mathrm{C}(26)-\mathrm{C}(29)-\mathrm{H}(29 \mathrm{~B})$ & 109.5 \\
\hline$H(29 A)-C(29)-H(29 B)$ & 109.5 \\
\hline $\mathrm{C}(26)-\mathrm{C}(29)-\mathrm{H}(29 \mathrm{C})$ & 109.5 \\
\hline$H(29 A)-C(29)-H(29 C)$ & 109.5 \\
\hline$H(29 B)-C(29)-H(29 C)$ & 109.5 \\
\hline$C(32)-C(31)-C(36)$ & $117.4(3)$ \\
\hline$C(32)-C(31)-C(4)$ & $123.3(3)$ \\
\hline$C(36)-C(31)-C(4)$ & $119.3(3)$ \\
\hline$C(33)-C(32)-C(31)$ & $120.9(3)$ \\
\hline $\mathrm{C}(33)-\mathrm{C}(32)-\mathrm{H}(32)$ & 119.6 \\
\hline$C(31)-C(32)-H(32)$ & 119.6 \\
\hline$C(34)-C(33)-C(32)$ & $120.3(4)$ \\
\hline $\mathrm{C}(34)-\mathrm{C}(33)-\mathrm{H}(33)$ & 119.9 \\
\hline $\mathrm{C}(32)-\mathrm{C}(33)-\mathrm{H}(33)$ & 119.9 \\
\hline$C(33)-C(34)-C(35)$ & $120.8(4)$ \\
\hline $\mathrm{C}(33)-\mathrm{C}(34)-\mathrm{H}(34)$ & 119.6 \\
\hline$C(35)-C(34)-H(34)$ & 119.6 \\
\hline$C(36)-C(35)-C(34)$ & $118.1(3)$ \\
\hline$C(36)-C(35)-H(35)$ & 120.9 \\
\hline$C(34)-C(35)-H(35)$ & 120.9 \\
\hline$O(1)-C(36)-C(35)$ & $125.0(3)$ \\
\hline$O(1)-C(36)-C(31)$ & $112.4(3)$ \\
\hline$C(35)-C(36)-C(31)$ & $122.6(3)$ \\
\hline $\mathrm{C}(97)-\mathrm{C}(96)-\mathrm{H}(96 \mathrm{~A})$ & 109.5 \\
\hline $\mathrm{C}(97)-\mathrm{C}(96)-\mathrm{H}(96 \mathrm{~B})$ & 109.5 \\
\hline $\mathrm{H}(96 \mathrm{~A})-\mathrm{C}(96)-\mathrm{H}(96 \mathrm{~B})$ & 109.5 \\
\hline $\mathrm{C}(97)-\mathrm{C}(96)-\mathrm{H}(96 \mathrm{C})$ & 109.5 \\
\hline $\mathrm{H}(96 \mathrm{~A})-\mathrm{C}(96)-\mathrm{H}(96 \mathrm{C})$ & 109.5 \\
\hline $\mathrm{H}(96 \mathrm{~B})-\mathrm{C}(96)-\mathrm{H}(96 \mathrm{C})$ & 109.5 \\
\hline$C(96)-C(97)-0(98)$ & $106.2(5)$ \\
\hline $\mathrm{C}(96)-\mathrm{C}(97)-\mathrm{H}(97 \mathrm{~A})$ & 110.5 \\
\hline $\mathrm{O}(98)-\mathrm{C}(97)-\mathrm{H}(97 \mathrm{~A})$ & 110.5 \\
\hline $\mathrm{C}(96)-\mathrm{C}(97)-\mathrm{H}(97 \mathrm{~B})$ & 110.5 \\
\hline $\mathrm{O}(98)-\mathrm{C}(97)-\mathrm{H}(97 \mathrm{~B})$ & 110.5 \\
\hline $\mathrm{H}(97 \mathrm{~A})-\mathrm{C}(97)-\mathrm{H}(97 \mathrm{~B})$ & 108.7 \\
\hline$O(98)-C(98)-C(99)$ & $110.1(10)$ \\
\hline $\mathrm{O}(98)-\mathrm{C}(98)-\mathrm{C}\left(99^{\prime}\right)$ & $110.7(11)$ \\
\hline$C(99)-C(98)-C\left(99^{\prime}\right)$ & $82.7(10)$ \\
\hline $\mathrm{O}(98)-\mathrm{C}(98)-0(99)$ & $114.0(7)$ \\
\hline$C(99)-C(98)-0(99)$ & $109.5(10)$ \\
\hline$C\left(99^{\prime}\right)-C(98)-0(99)$ & $124.9(10)$ \\
\hline $\mathrm{C}(98)-\mathrm{C}(99)-\mathrm{H}(99 \mathrm{~A})$ & 109.5 \\
\hline $\mathrm{C}(98)-\mathrm{C}(99)-\mathrm{H}(99 \mathrm{~B})$ & 109.5 \\
\hline $\mathrm{C}(98)-\mathrm{C}(99)-\mathrm{H}(99 \mathrm{C})$ & 109.5 \\
\hline $\mathrm{C}(98)-\mathrm{C}\left(99^{\prime}\right)-\mathrm{H}(99 \mathrm{D})$ & 109.5 \\
\hline $\mathrm{C}(98)-\mathrm{C}\left(99^{\prime}\right)-\mathrm{H}(99 \mathrm{E})$ & 109.5 \\
\hline $\mathrm{H}(99 \mathrm{D})-\mathrm{C}\left(99^{\prime}\right)-\mathrm{H}(99 \mathrm{E})$ & 109.5 \\
\hline $\mathrm{C}(98)-\mathrm{C}\left(99^{\prime}\right)-\mathrm{H}(99 \mathrm{~F})$ & 109.5 \\
\hline
\end{tabular}

Supplementary data for $10.1021 / \mathrm{ja} 063186 \mathrm{w}$ 


$\begin{array}{ll}\mathrm{H}(99 \mathrm{D})-\mathrm{C}\left(99^{\prime}\right)-\mathrm{H}(99 \mathrm{~F}) & 109.5 \\ \mathrm{H}(99 \mathrm{E})-\mathrm{C}\left(99^{\prime}\right)-\mathrm{H}(99 \mathrm{~F}) & 109.5 \\ \mathrm{C}(1)-\mathrm{N}(1)-\mathrm{C}(21) & 128.5(3) \\ \mathrm{C}(1)-\mathrm{N}(1)-\mathrm{C}(2) & 114.4(3) \\ \mathrm{C}(21)-\mathrm{N}(1)-\mathrm{C}(2) & 117.0(3) \\ \mathrm{C}(1)-\mathrm{N}(2)-\mathrm{C}(11) & 128.1(3) \\ \mathrm{C}(1)-\mathrm{N}(2)-\mathrm{C}(3) & 114.3(3) \\ \mathrm{C}(11)-\mathrm{N}(2)-\mathrm{C}(3) & 116.0(3) \\ \mathrm{C}(36)-\mathrm{O}(1)-\mathrm{C}(5) & 121.8(3) \\ \mathrm{C}(36)-\mathrm{O}(1)-\mathrm{Ru}(1) & 110.39(19) \\ \mathrm{C}(5)-\mathrm{O}(1)-\mathrm{Ru}(1) & 126.0(2) \\ \mathrm{C}(6)-\mathrm{O}(3)-\mathrm{C}(7) & 115.8(3) \\ \mathrm{C}(98)-\mathrm{O}(98)-\mathrm{C}(97) & 120.7(6) \\ \mathrm{C}(4)-\mathrm{Ru}(1)-\mathrm{C}(1) & 100.47(14) \\ \mathrm{C}(4)-\mathrm{Ru}(1)-\mathrm{O}(1) & 81.19(12) \\ \mathrm{C}(1)-\mathrm{Ru}(1)-\mathrm{O}(1) & 176.56(12) \\ \mathrm{C}(4)-\mathrm{Ru}(1)-\mathrm{Cl}(2) & 97.75(12) \\ \mathrm{C}(1)-\mathrm{Ru}(1)-\mathrm{Cl}(2) & 94.97(10) \\ \mathrm{O}(1)-\mathrm{Ru}(1)-\mathrm{Cl}(2) & 87.76(7) \\ \mathrm{C}(4)-\mathrm{Ru}(1)-\mathrm{Cl}(1) & 94.67(12) \\ \mathrm{C}(1)-\mathrm{Ru}(1)-\mathrm{Cl}(1) & 91.61(10) \\ \mathrm{O}(1)-\mathrm{Ru}(1)-\mathrm{Cl}(1) & 85.24(7) \\ \mathrm{Cl}(2)-\mathrm{Ru}(1)-\mathrm{Cl}(1) & 164.63(3) \\ & \end{array}$

Symmetry transformations used to generate equivalent atoms:

Table 4. Anisotropic displacement parameters (?2x 103) for mb041004. The anisotropic displacement factor exponent takes the form:

$-2 \mathrm{p} 2[\mathrm{~h} 2 \mathrm{a} * 2 \mathrm{U} 11+\ldots+2 \mathrm{~h} \mathrm{k}$ a $* \mathrm{~b} * \mathrm{U} 12]$

\begin{tabular}{|c|c|c|c|c|c|c|}
\hline & U11 & U22 & U33 & U23 & U13 & U12 \\
\hline$C(1)$ & $20(2)$ & $18(2)$ & $19(2)$ & $-5(2)$ & $6(2)$ & $-2(2)$ \\
\hline$C(2)$ & $32(2)$ & $20(2)$ & $31(2)$ & $5(2)$ & $2(2)$ & $1(2)$ \\
\hline$C(3)$ & $33(2)$ & $18(2)$ & $31(2)$ & $7(2)$ & $4(2)$ & $1(2)$ \\
\hline$C(4)$ & $27(2)$ & $22(2)$ & $19(2)$ & $7(2)$ & $0(2)$ & $1(2)$ \\
\hline$C(5)$ & $23(2)$ & $27(2)$ & $25(2)$ & $6(2)$ & $2(2)$ & $4(2)$ \\
\hline$C(6)$ & $27(2)$ & $37(3)$ & $20(2)$ & $3(2)$ & $O(2)$ & $2(2)$ \\
\hline$C(7)$ & $35(3)$ & $58(3)$ & $89(4)$ & $-2(3)$ & $-3(3)$ & $-21(3)$ \\
\hline$C(8)$ & $35(3)$ & $39(3)$ & $54(3)$ & $16(2)$ & $18(2)$ & $13(2)$ \\
\hline$C(11)$ & $21(2)$ & $18(2)$ & $29(2)$ & $4(2)$ & $0(2)$ & $0(2)$ \\
\hline$C(12)$ & $26(2)$ & $23(2)$ & $36(2)$ & $4(2)$ & $-3(2)$ & $2(2)$ \\
\hline$C(13)$ & $24(2)$ & $43(3)$ & $58(3)$ & $2(2)$ & $2(2)$ & $2(2)$ \\
\hline$C(14)$ & $38(3)$ & $37(3)$ & $59(3)$ & $3(2)$ & $-13(2)$ & $-8(2)$ \\
\hline$C(15)$ & $59(3)$ & $26(3)$ & $30(2)$ & $3(2)$ & $-14(2)$ & $-2(2)$ \\
\hline$C(16)$ & $36(2)$ & $19(2)$ & $31(2)$ & $2(2)$ & $-2(2)$ & $5(2)$ \\
\hline$C(17)$ & $28(2)$ & $39(3)$ & $46(3)$ & $2(2)$ & $13(2)$ & $5(2)$ \\
\hline$C(18)$ & $52(3)$ & $81(5)$ & $91(4)$ & $-16(4)$ & $-23(3)$ & $-22(3)$ \\
\hline$C(19)$ & $63(3)$ & $37(3)$ & $29(2)$ & $1(2)$ & $11(2)$ & $4(2)$ \\
\hline
\end{tabular}

Supplementary data for $10.1021 / \mathrm{ja} 063186 \mathrm{w}$ 


$\begin{array}{lllllll}\mathrm{C}(21) & 28(2) & 19(2) & 26(2) & 4(2) & 5(2) & -3(2) \\ \mathrm{C}(22) & 40(3) & 25(2) & 36(3) & 7(2) & 19(2) & 3(2) \\ \mathrm{C}(23) & 36(3) & 45(3) & 65(4) & 15(3) & 26(2) & 12(2) \\ \mathrm{C}(24) & 33(3) & 46(3) & 65(4) & 14(3) & -4(3) & -1(2) \\ \mathrm{C}(25) & 37(3) & 38(3) & 40(3) & 3(2) & -6(2) & 0(2) \\ \mathrm{C}(26) & 32(2) & 16(2) & 29(2) & 4(2) & 4(2) & 0(2) \\ \mathrm{C}(27) & 70(3) & 42(3) & 34(3) & -1(2) & 25(2) & 2(2) \\ \mathrm{C}(28) & 25(3) & 125(6) & 144(6) & 36(5) & 2(3) & 1(3) \\ \mathrm{C}(29) & 41(3) & 27(2) & 28(2) & -4(2) & 4(2) & -2(2) \\ \mathrm{C}(31) & 25(2) & 18(2) & 21(2) & -1(2) & 2(2) & 0(2) \\ \mathrm{C}(32) & 36(2) & 23(2) & 30(2) & 0(2) & -6(2) & 3(2) \\ \mathrm{C}(33) & 33(2) & 22(2) & 47(3) & -2(2) & 0(2) & -7(2) \\ \mathrm{C}(34) & 45(3) & 20(2) & 41(3) & 8(2) & 10(2) & -3(2) \\ \mathrm{C}(35) & 34(2) & 23(2) & 27(2) & 4(2) & 6(2) & 4(2) \\ \mathrm{C}(36) & 25(2) & 18(2) & 19(2) & 1(2) & 1(2) & 1(2) \\ \mathrm{C}(96) & 74(4) & 101(5) & 84(5) & 19(5) & 4(3) & 11(4) \\ \mathrm{C}(97) & 78(5) & 67(4) & 83(5) & -12(4) & 5(4) & 11(3) \\ \mathrm{C}(98) & 54(5) & 101(7) & 215(11) & -68(8) & 3(6) & -6(5) \\ \mathrm{O}(99) & 105(4) & 250(8) & 202(6) & -40(6) & 12(4) & -20(4) \\ \mathrm{C}(99) & 34(7) & 88(10) & 157(15) & -67(11) & -2(8) & -1(6) \\ \mathrm{C}\left(99^{\prime}\right) & 71(9) & 85(10) & 131(14) & 65(10) & -6(9) & -16(7) \\ \mathrm{N}(1) & 23(2) & 22(2) & 20(2) & 5(1) & 2(1) & -3(1) \\ \mathrm{N}(2) & 25(2) & 16(2) & 27(2) & 6(1) & 5(1) & -1(1) \\ \mathrm{O}(1) & 23(1) & 17(1) & 33(2) & 4(1) & -4(1) & -1(1) \\ \mathrm{O}(2) & 39(2) & 34(2) & 42(2) & 3(2) & 4(1) & -2(2) \\ \mathrm{O}(3) & 32(2) & 48(2) & 69(2) & 12(2) & -3(2) & -3(2) \\ \mathrm{O}(98) & 75(3) & 101(4) & 130(4) & -17(3) & -23(3) & -12(3) \\ \mathrm{C} 1(1) & 35(1) & 29(1) & 21(1) & -1(1) & 6(1) & 3(1) \\ \mathrm{C}(2) & 41(1) & 33(1) & 28(1) & 0(1) & 13(1) & 7(1) \\ \mathrm{Ru}(1) & 25(1) & 17(1) & 19(1) & 0(1) & 3(1) & 1(1)\end{array}$

